BULLETIN (New Series) OF THE

AMERICAN MATHEMATICAL SOCIETY

Volume 36, Number 3, Pages 271-296

S 0273-0979(99)00798-3

Article electronically published on June 24, 1999

\title{
STRONG SHIFT EQUIVALENCE THEORY AND THE SHIFT EQUIVALENCE PROBLEM
}

\author{
J. B. WAGONER
}

\begin{abstract}
This paper discusses strong shift equivalence and counterexamples to the long standing Shift Equivalence Problem in symbolic dynamics. We also discuss how strong shift equivalence theory is closely related to areas of mathematics outside dynamics such as algebraic K-theory, cyclic homology, and topological quantum field theory.
\end{abstract}

\section{INTRODUCTION}

Subshifts of finite type arise in a wide range of subjects from dynamical systems to statistical mechanics, to $C^{*}$-algebras, to coding and information theory. We will later define subshifts of finite type along with other terms appearing in this section, and we will explain the topological classification problem. We will also explain how strong shift equivalence theory grew out of R.F.Williams' fundamental work [Wi1] in which he formulated an algebraic approach to the classification problem by introducing strong shift equivalence and shift equivalence over the nonnegative integers $Z^{+}$, the latter being much more accessible algebraically. These concepts can be expressed in an elementary fashion by graphs and matrices. Whether shift equivalence implies strong shift equivalence over $Z^{+}$has been a well known and tantalizing question since 1974, called the Shift Equivalence Problem. In 1997 a counterexample to this conjecture was finally found for primitive matrices by KimRoush in [KR5], [KR6] using the sign-gyration-compatibility-condition cohomology class $s g c_{k}$ for $k=2$ arising from a construction in [KRW1]. Subsequently in [W7] another primitive counterexample similar to that of Kim-Roush was found by using an invariant $\Phi_{2 m}$ in the algebraic K-theory group $K_{2}$ of the dual numbers for $m=1$. The one-dimensional cohomology classes $s g c_{k}$ were constructed by studying how symmetries of shifts act on periodic points. On the other hand, $\Phi_{2 m}$ comes from a very different construction using an analogy with pseudo-isotopy theory. Numerical evidence suggested that $s g c_{2}=\Phi_{2}$, and this was proved by Kim-Roush in the Appendix to [W7]. While strong shift equivalence theory had its origin in dynamics, it turns out to be closely related to other areas such as number theory, algebraic K-theory, and cyclic homology. In addition, Gilmer [G2] has recently shown there is a connection between topological quantum field theory and strong shift equivalence over $Z^{+}$. Related work has been done by D. Silver and S. Williams

Received by the editors April 29, 1999, and in revised form May 27, 1999.

1991 Mathematics Subject Classification. Primary 19C99, 19D55, 58F99, 81R99.

Key words and phrases. Strong shift equivalence, the shift equivalence problem, positivity.

The author was supported in part by NSF Grant DMS 9322498.

(C)1999 American Mathematical Society 
[SW1], [SW2] in the context of combinatorial group theory. This article surveys some of these developments.

Section 2 presents basic material about subshifts of finite type and gives the precise statement of the Shift Equivalence Problem. Section 3 defines the strong shift equivalence spaces. Section 4 presents the $\Delta$-strategy for discussing the difference between shift equivalence and strong shift equivalence. Section 5 gives a specific counterexample to the Shift Equivalence Problem which is detected by $\Delta=s g c_{2}=\Phi_{2}$. Section 6 and Section 7 outline the construction of $s g c_{k}$ and $\Phi_{2 m}$ respectively. Section 8 discusses strong shift equivalence and topological quantum field theory. Section 9 presents further projects and problems. Good general background references are $[\mathrm{K}],[\mathrm{LM}],[\mathrm{R}]$. A sampling of articles is $[\mathrm{A}],[\mathrm{BH} 2]$, [CK2], $[\mathrm{E}],[\mathrm{G} 2],[\mathrm{M}],[\mathrm{S}]$.

\section{Subshifts of Finite type AND the ClassificAtion PROBlem}

The full Bernoulli n-shift is the set $X_{n}$ of bi-infinite sequences $x=\left\{x_{k}\right\}$ where $x_{k}$ is a symbol taken from a set $S$ of cardinality $n$. Typically, one takes $S=$ $\{0, \ldots, n-1\}$. We give $X_{n}$ the product topology, making it a Cantor set. The shift homeomorphism $\sigma_{n}: X_{n} \rightarrow X_{n}$ is defined by $\sigma_{n}(x)_{k}=x_{k+1}$. In other words, the shift moves each sequence to the left one step. A block is a sequence $\left[s_{1}, s_{2}, \ldots, s_{p}\right]$ of finite length where each $s_{i}$ is in $S$. A subshift of finite type arises by fixing a finite set $F$ of blocks and then excluding from $X_{n}$ all $x$ which contain a block in $F$. For example, if $F$ consists of the single block [11], then we obtain the golden mean shift consisting of those sequences in $X_{2}$ such that such a 0 can be followed on the right by either a 0 or a 1 , but a 1 must be followed by a 0 . Interesting and very practical examples are the run length limited subshifts used in data storage as discussed in $[\mathrm{A}],[\mathrm{LM}]$. Magnetic storage devices are based on algorithms that can code an arbitrary sequence of zeros and ones into another sequence of zeros and ones which has some constraints due to practical considerations. Consider the subshift $L(2,7)$ of $X_{2}$ which consists of all those $x$ which have infinitely many ones and which have at least 2 but not more than 7 zeros between any two successive ones. This models a magnetic tape streaming along, with 1 being the symbol for reversal of magnetic fields and 0 being the symbol for nonreversal. Insisting upon at least two zeros helps prevent intersymbol interference or confusion of magnetic fields. The bound of seven zeros helps maintain accuracy of a clock, which is updated at each reversal.

An equivalent way to obtain a subshift of finite type is to consider an $m \times m$ zero-one transition matrix $A$. This determines the subshift of finite type $\left\{X_{A}, \sigma_{A}\right\}$ by letting $X_{A}$ be the subset of sequences $x$ in $X_{m}$ satisfying $A\left(x_{k}, x_{k+1}\right)=1$ for all $-\infty<k<\infty$. By definition, $\sigma_{A}=\sigma_{m} \mid X_{A}$. The $m \times m$ matrix of all 1's gives the full Bernoulli $m$-shift. More generally, the standard edge shift construction [LM] allows one to obtain a subshift of finite type $\left(X_{A}, \sigma_{A}\right)$ from any $m \times m$ nonnegative integral matrix $A=\left\{A_{i j}\right\}$. The matrix $A$ can be viewed as a directed graph which has $m$ vertices and which has $A_{i j}$ edges going from the vertex $i$ to the vertex $j$. Order the set $S^{\#}$ of edges of the graph $A$ and define the zero-one matrix $A^{\#}: S^{\#} \times S^{\#} \rightarrow\{0,1\}$ by letting $A^{\#}(\alpha, \beta)=1$ iff the end vertex of the edge $\alpha$ is the start vertex of the edge $\beta$. Then one defines

$$
\left(X_{A}, \sigma_{A}\right)=\left\{X_{A^{\#}}, \sigma_{A^{\#}}\right\} .
$$


This is a subshift of the full Bernoulli $m^{\#}$-shift where $m^{\#}$ is the number of edges of $A$. For example, $\left(X_{2}, \sigma_{2}\right)=\left(X_{A}, \sigma_{A}\right)$ where $A=\{2\}$, because $A^{\#}$ is the $2 \times 2$ matrix of all 1's. If $A$ is a zero-one matrix, the shifts $\left\{X_{A}, \sigma_{A}\right\}$ and $\left\{X_{A^{\#}}, \sigma_{A^{\#}}\right\}$ are canonically equivalent as explained in [W4].

One way in which subshifts of finite type arise in dynamical systems is through Markov partitions, which were a key feature in the development of dynamical system theory in the 1960's. Smale's influential article [S] summarized how Markov partitions could be used to study the recurrent behaviour of discrete time dynamical systems and continuous flows. See [A] and [LM, Section 6.5] also. Let $f: X \rightarrow X$ be a homeomorphism (i.e., a reversible discrete dynamical system). For example, $X$ could be the zero-dimensional hyperbolic nonwandering set of a diffeomorphism on a higher dimensional manifold. A Markov partition is a way of writing this zero-dimensional $X$ as a disjoint union of open sets

$$
X=U_{1} \cup U_{2} \cup \ldots \cup U_{n}
$$

which satisfy certain axioms. See $[\mathrm{LM}],[\mathrm{PT}]$ and (3.12) below. Define the zero-one matrix $A$ by the condition that

$$
A(i, j)=1 \text { iff } U_{i} \cap f^{-1} U_{j} \text { is not empty. }
$$

Define $\pi: X \rightarrow X_{n}$ by letting $\pi(x)_{k}=i$ iff $f^{k}(x)$ is in $U_{i}$. Then $\pi$ is a homeomorphism from $X$ to $X_{A}$, and moreover $\pi f=\sigma_{A} \pi$. Thus $\pi$ is a topological conjugacy between the dynamical systems $(X, f)$ and $\left\{X_{A}, \sigma_{A}\right\}$.

Dynamical systems which are topologically conjugate have the same underlying behaviour. In this regard subshifts of finite type are systems where it is relatively easy to explicitly compute dynamically intrinsic quantities. See [LM]. One of the most important and basic invariant quantities is the topological entropy $h(f)$ defined by Adler-Konheim-McAndrew in [AKM]. This is a topological version of entropy in ergodic theory [Kol] and channel capacity in information theory [Sh]. When $X=X_{A}$ and $f=\sigma_{A}$, the topological entropy $h\left(\sigma_{A}\right)$ can be computed by the formula

$$
h\left(\sigma_{A}\right)=\lim _{n \rightarrow \infty} \frac{1}{n} \log B_{n}=\log \lambda
$$

where $B_{n}$ is the number of blocks of length $n$ appearing in sequences of $X_{A}$ and where $\lambda=\lambda_{A}$ is the Perron-Frobenius eigenvalue, the largest real root of $\operatorname{det}(t I-A)$. See [LM, Section 4]. Another important invariant is the Artin-Mazur zeta function which elegantly combines information about the number of periodic points. For a subshift of finite type it is given by the formula

$$
\zeta_{A}(t)=\exp \left(\sum_{n=1}^{\infty} \frac{p_{n}\left(\sigma_{A}\right)}{n} t^{n}\right)
$$

where $p_{n}\left(\sigma_{A}\right)$ is the number of periodic points $x$ in $X_{A}$ such that $\sigma_{A}^{n}(x)=x$. In fact, $p_{n}\left(\sigma_{A}\right)=\operatorname{Tr}\left(A^{n}\right)$, and there is the Bowen-Lanford formula [LM, 6.4.6] which says that

$$
\zeta_{A}(t)=\frac{1}{\operatorname{det}(I-t A)}
$$

When a Markov partition can be shown to exist [Bow] and its structure is clear enough to read off the transition matrix $[\mathrm{A}],[\mathrm{LM}],[\mathrm{S}]$, it can be used to obtain information about the dynamical system in question such as entropy and the zeta 

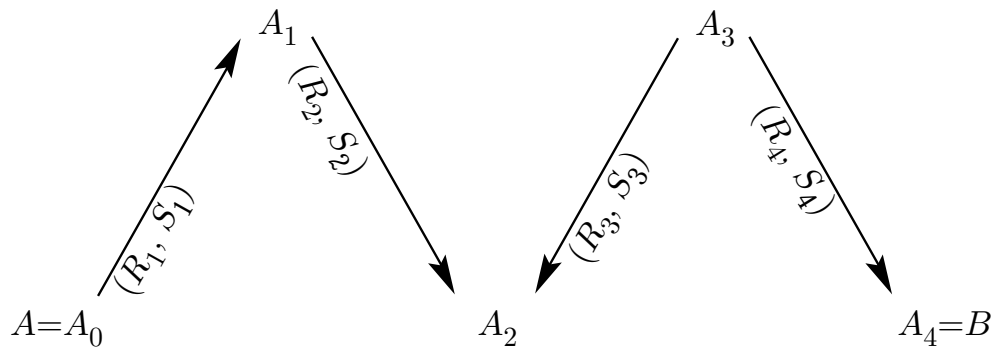

FiguRE 1

function. However, if one Markov partition exists, then there are infinitely many. In other words, there are typically infinitely many ways to construct or present dynamical systems that have equivalent behaviour. So there is the fundamental and still open

Classification Problem. Given nonnegative integral matrices $A$ and $B$, when are the corresponding subshifts of finite type $\left(X_{A}, \sigma_{A}\right)$ and $\left(X_{B}, \sigma_{B}\right)$ topologically conjugate?

R.F. Williams formulated an algebraic approach to this question, which we now review. An elementary strong shift equivalence $(R, S): M \rightarrow N$ over the nonnegative integers $Z^{+}$consists of two nonnegative integral matrices of finite size $R$ and $S$ satisfying the strong shift equivalence equations

$$
M=R S, \quad S R=N .
$$

Williams defined the matrices $A$ and $B$ to be strong shift equivalent over $Z^{+}$iff there is a chain of elementary strong shift equivalences over $Z^{+}$between them. For example, a chain of length 4 is illustrated by Figure 1 . We shall see below that $R$ and $S$ play different roles, so the direction of the arrow in the notation $(R, S): M \rightarrow N$ is important. In [Wi1] Williams proved

Theorem 2.4. $\left(X_{A}, \sigma_{A}\right)$ and $\left(X_{B}, \sigma_{B}\right)$ are topologically conjugate iff $A$ and $B$ are strong shift equivalent over $Z^{+}$.

Also see $[\mathrm{Fr}],[\mathrm{LM}],[\mathrm{W} 1]$ for expositions of this result. Here is an example. Let

$$
\begin{aligned}
& A=\left(\begin{array}{ll}
1 & 1 \\
1 & 1
\end{array}\right) \quad B=\left(\begin{array}{lll}
1 & 1 & 0 \\
0 & 0 & 1 \\
1 & 1 & 1
\end{array}\right) \\
& R=\left(\begin{array}{lll}
1 & 1 & 0 \\
0 & 0 & 1
\end{array}\right) \quad S=\left(\begin{array}{ll}
1 & 0 \\
0 & 1 \\
1 & 1
\end{array}\right) .
\end{aligned}
$$

Observe that $\left\{X_{A}, \sigma_{A}\right\}=\left(X_{2}, \sigma_{2}\right)$ and that $\left\{X_{B}, \sigma_{B}\right\}$ is a subshift of $\left(X_{3}, \sigma_{3}\right)$. We have $A=R S$ and $S R=B$, and the strong shift equivalence $(R, S): A \rightarrow B$ produces a topological conjugacy

$$
c(R, S):\left\{X_{A}, \sigma_{A}\right\} \rightarrow\left\{X_{B}, \sigma_{B}\right\}
$$


as follows. Let $x=\left\{x_{k}\right\}$ and $y=\left\{y_{k}\right\}$ where $y=c(R, S)(x)$. We have

$$
1=A\left(x_{k}, x_{k+1}\right)=\sum_{i} R\left(x_{k}, i\right) S\left(i, x_{k+1}\right)
$$

Since $A, R$, and $S$ are zero-one matrices, there is exactly one $i$ for which $R\left(x_{k}, i\right)=$ $S\left(i, x_{k+1}\right)=1$. By definition, $y_{k}=i$. We have $c(R, S) c(S, R)=c(A, 1)=\sigma_{A}$ and $c(S, R) c(R, S)=c(B, 1)=\sigma_{B}$. Here the composition is read from left to right. This process generalizes to matrices over $Z^{+}$using the \#-construction. See [LM, 7.2] and [W4].

Strong shift equivalence over $Z^{+}$is difficult to determine. A more algebraically tractable concept is shift equivalence. The matrices $A$ and $B$ are said to be shift equivalent over $Z^{+}$iff there are nonnegative matrices $R$ and $S$ and a positive integer $k$, called the lag, satisfying the shift equivalence equations

$$
A R=R B, \quad B S=S A, \quad A^{k}=R S, \quad S R=B^{k} .
$$

Two subshifts of finite type $\left(X_{A}, \sigma_{A}\right)$ and $\left(X_{B}, \sigma_{B}\right)$ are eventually conjugate if there is an integer $N$ such that $\left(X_{A}, \sigma_{A}^{k}\right)$ and $\left(X_{B}, \sigma_{B}^{k}\right)$ are topologically conjugate for $k \geq N$. Williams [Wi1] and Kim-Roush [KR1] proved

Theorem 2.6. $\left(X_{A}, \sigma_{A}\right)$ and $\left(X_{B}, \sigma_{B}\right)$ are eventually conjugate iff $A$ and $B$ are shift equivalent over $Z^{+}$.

See [LM, 7.5.15]. Obviously, conjugacy implies eventual conjugacy and strong shift equivalence implies shift equivalence. Williams' influential work [Wi1] brought forth the following question.

Shift Equivalence Problem 2.7. Does SE over $Z^{+}$imply SSE over $Z^{+}$?

Shift equivalence over $Z^{+}$is much easier to determine than strong shift equivalence over $Z^{+}$. Shift equivalence is decidable [KR1], [KR2], and Krieger characterized shift equivalence in terms of the dimension group triple $\left(G_{A}, G_{A}^{+}, s_{A}\right)$. See [LM, 7.5]. The most concrete definition of $G_{A}$ uses the eventual range $R_{A}$. Suppose $A$ is an $n \times n$ matrix, and consider it as a linear transformation from $Q^{n}$ to itself. We let $R_{A}=A^{m}\left(Q^{n}\right)$ where $m$ is sufficiently large that $A: A^{m}\left(Q^{n}\right) \rightarrow A^{m+1}\left(Q^{n}\right)$ is an isomorphism. Then

$$
\begin{aligned}
& G_{A}=\left\{v \mid v \epsilon R_{A} \text { and } v A^{p} \epsilon Z^{n} \text { for some integer } p \geq 0\right\} \\
& G_{A}^{+}=\left\{v \mid v \epsilon G_{A} \text { and } v A^{p} \geq 0 \text { for some integer } p \geq 0\right\} \\
& s_{A} \text { is the isomorphism of } G_{A} \text { induced by } A .
\end{aligned}
$$

Example 2.9. $A=\{2\}$. Then $G_{A}=Z\left[\frac{1}{2}\right], G_{A}=Z\left[\frac{1}{2}\right]^{+}$, and $s_{A}$ is multiplication by 2 .

Example 2.10. Consider the golden mean shift defined by the matrix

$$
A=\left(\begin{array}{ll}
1 & 1 \\
1 & 0
\end{array}\right)
$$

Since $\operatorname{det}(A)=-1$, we have $G_{A}=Z^{2}$. The characteristic polynomial of $A$ is $t^{2}-t-1$, and the Perron-Frobenius eigenvalue $\lambda$ satisfies the equation $\lambda^{2}=\lambda+1$. The vector $(\lambda, 1)$ is a right eigenvector for $\lambda$. The correspondence sending the pair of integers $(a, b)$ to $a \lambda+b$ induces an isomorphism between $G_{A}$ and $Z[\lambda]$, considered as a subgroup of the real numbers $R$ equipped with its usual ordering. Via this isomorphism, $s_{A}$ corresponds to multiplication by $\lambda$ and $G_{A}^{+}$becomes $Z[\lambda]^{+}$. 
The name "dimension group" comes from operator algebra theory where it is possible to have dimension functions on the projections in infinite dimensional algebras which may take on nonintegral real number values. In particular, a nonnegative integral matrix $A$ gives rise to a certain $\mathrm{C}^{*}$-algebra via a Bratelli diagram so that the range of the dimension function is $G_{A}$. See [E], [W6] for expositions of these ideas.

Krieger's theorem [LM, 7.5.8] states

Theorem 2.11. Nonnegative integral matrices $A$ and $B$ are shift equivalent over $Z^{+}$iff $\left(G_{A}, G_{A}^{+}, s_{A}\right)$ is isomorphic to $\left(G_{B}, G_{B}^{+}, s_{B}\right)$.

The most important case is when $A$ and $B$ are primitive. This means there is a positive integer $m$ such that both $A^{m}$ and $B^{m}$ have all entries positive. The dynamical significance is that the subshifts $\left(X_{A}, \sigma_{A}\right)$ and $\left(X_{B}, \sigma_{B}\right)$ are topologically mixing [LM, Section 6.3]. It turns out that primitive matrices are shift equivalent over $Z^{+}$iff they are shift equivalent over $Z$ [LM, 7.5]. This implies that primitive $A$ and $B$ are shift equivalent over $Z^{+}$iff the dimension group pairs $\left(G_{A}, s_{A}\right)$ and $\left(G_{B}, s_{B}\right)$ are isomorphic. See [LM, 7.5.9]. In this paper, we will mainly consider $A$ such that $\operatorname{det}(A)= \pm 1$, and then there is the very concrete description

$$
G_{A}=Z^{n}, \quad s_{A}=\text { the isomorphism of } G_{A} \text { given by } A .
$$

Thus Krieger's Theorem and the above discussion yield

Corollary 2.13. Suppose $\operatorname{det}(A)= \pm 1$ and $\operatorname{det}(B)= \pm 1$. Then $A$ and $B$ are shift equivalent over $Z^{+}$iff the matrices $A$ and $B$ are conjugate in $G l_{n}(Z)$.

In certain categories SE does imply SSE. Effros-Williams showed that SE over $Z$ implies SSE over $Z$. For an exposition of this see [W3] or see [BH1] where a proof is given that SE implies SSE for Dedekind domains $\Lambda$. A key step in the argument when $\Lambda$ is a principal ideal domain, say $\Lambda=Z$, is the following: Consider an $m \times n$ integer matrix $A$ as a homomorphism $A: Z^{m} \rightarrow Z^{n}$. Write $A=R S$ where $R$ is the map $A$ from $Z^{m}$ to $\operatorname{Image}(A)$ and $S$ is the inclusion of $\operatorname{Image}(A)$ into $Z^{n}$. Since $Z$ is a principal ideal domain, Image $(A)$ is a free, finitely generated $Z$-submodule. Choose a finite basis for it. With respect to this basis and the standard bases for $Z^{m}$ and $Z^{n}$, the equation $A=R S$ becomes a matrix equation. The difficulty in using the above argument to show SE implies SSE over $Z^{+}$is that it may not be possible to choose the basis for Image $(A)$ so that both $R$ and $S$ are nonnegative. A similar argument appears in the proof that SE implies SSE for endomorphisms of finitely generated free groups. In addition, Williams [Wi3], [Wi4] proved that SE implies SSE for positive endomorphisms of free groups. In general, there is the

Algebraic Shift Equivalence Problem 2.14. For what rings $\Lambda$ does $\mathrm{SE}$ over $\Lambda$ imply $\operatorname{SSE}$ over $\Lambda$ ?

In any case, when $\Lambda=Z$ we know that SE over $\Lambda^{+}$is equivalent to SSE over $\Lambda$ for primitive matrices. So an alternative formulation of the Shift Equivalence Problem over $Z^{+}$is therefore

Strong Shift Equivalence Problem 2.15. If $A$ and $B$ are primitive nonnegative integral matrices, does SSE over $Z$ imply SSE over $Z^{+}$? 
The answer is sometimes "yes" for $2 \times 2$ matrices [B], [CK1], [Wi2], although here is a well known example where the question is still open. Let

$$
A_{n}=\left(\begin{array}{cc}
1 & n(n-1) \\
1 & 1
\end{array}\right) \quad B_{n}=\left(\begin{array}{cc}
1 & n \\
n-1 & 1
\end{array}\right) \quad R_{n}=\left(\begin{array}{cc}
n-1 & n \\
1 & 1
\end{array}\right)
$$

Then $\operatorname{det}\left(R_{n}\right)=-1$ and $A_{n} R_{n}=R_{n} B_{n}$. So $A_{n}$ and $B_{n}$ are SSE over $Z$. Observe that $A_{2}=B_{2}$. Kirby Baker showed that $A_{3}$ is SSE to $B_{3}$ over $Z^{+}$. There is a chain of seven elementary SSE's between them, some of which involve $4 \times 4$ matrices. See [LM, p. 238]. Is $A_{n}$ SSE to $B_{n}$ over $Z^{+}$for $n \geq 4$ ? Kim-Roush have shown in [KR7], [KR8] that $A_{n}$ is SSE to $B_{n}$ over $Q^{+}$for $n \geq 2$.

A specific $7 \times 7$ counterexample to the Strong Shift Equivalence Problem similar to the first such example given in [KR5], [KR6] will be discussed in Section 5.

\section{Strong Shift equivalence SPACES}

A general program for studying the difference between SSE over $Z^{+}$and SSE over $Z$ involves the strong shift equivalence spaces $S S E\left(Z^{+}\right)$and $S S E(Z)$. These $\mathrm{CW}$ complexes arose in the study of automorphism groups of subshifts of finite type. See [BaW], [W1], [W2], [W3], [W4], [W5], [KRW1]. Previously, they were denoted by $R S\left(Z^{+}\right)$and $R S(Z)$ in the literature. We believe the $S S E$ notation is more appropriate. In this section we first give the definition of the $S S E$ spaces and state the main results about them. Then we discuss how they were found.

The vertices of $S S E\left(Z^{+}\right)$are finite, square matrices $A$ with nonnegative integer entries. The edges are elementary strong shift equivalences $(R, S): A \rightarrow B$ over $Z^{+}$. It is possible that $A=B$, in which case a loop is created at the vertex $A$. The 2-cells come from triangles

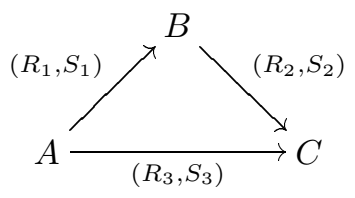

where the following Triangle Identities hold:

$$
R_{1} R_{2}=R_{3}, R_{2} S_{3}=S_{1}, S_{3} R_{1}=S_{2} .
$$

The precise definition of an n-cell of $S S E\left(Z^{+}\right)$is given in [W2], [W4] and in (9.5) below. The definition of $S S E(Z)$ is entirely similar, as is the definition of $S S E(Z O)$ which is formed by considering only matrices in the category $Z O$ of zero-one matrices. Ditto for $S S E\left(\Lambda^{+}\right)$and $S S E(\Lambda)$ for more general rings $\Lambda$. Strictly speaking, in forming $S S E(Z O)$ or $S S E\left(\Lambda^{+}\right)$we assume the technical condition that each row and each column of a vertex $A$ has a nonzero entry. The case when $\Lambda$ is a ring of integral Laurent polynomials arises from Markov chains. $\Lambda^{+}$consists of those Laurent polynomials with nonnegative coefficients. See [Bo], [MT1], [MT2], [MT3], $[\mathrm{T}]$. The case when $\Lambda$ is the group ring $Z[G]$ of a finite group $G$ arises from free actions of $G$ on subshifts of finite type. 


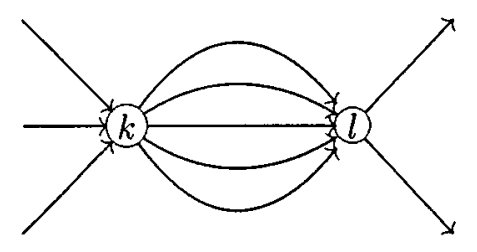

Figure 2

It follows from the definitions and Williams paper [Wi1] that

$$
\begin{aligned}
& \pi_{0}(S S E(Z O))=\text { strong shift equivalence classes over } Z O \\
& \pi_{0}\left(S S E\left(Z^{+}\right)\right)=\text {strong shift equivalence classes over } Z^{+} \\
& \pi_{0}(S S E(Z))=\text { strong shift equivalence classes over } Z \text {. }
\end{aligned}
$$

See [W4, 2.1] also.

The group $\operatorname{Aut}\left(\sigma_{A}\right)$ of automorphisms of the shift $\left(X_{A}, \sigma_{A}\right)$ consists of those homeomorphisms $f: X_{A} \rightarrow X_{A}$ which commute with $\sigma_{A}$. It was first studied by Hedlund and coworkers at IDA in the 1960's in connection with coding theory. See $[\mathrm{H}]$. In the 1980's there was a renewal of interest in $A u t\left(\sigma_{A}\right)$. See [BLR]. Clearly, $\sigma_{A}$ itself belongs to $A u t\left(\sigma_{A}\right)$. Any permutation $\alpha$ on $n$ symbols gives an element of $\operatorname{Aut}\left(\sigma_{n}\right)$ by simply permuting each symbol; i.e., $\alpha(x)_{k}=\alpha\left(x_{k}\right)$ for $x=\left\{x_{k}\right\}$. Aut $\left(\sigma_{n}\right)$ contains the direct sum of any countable set of finite groups as well as the direct sum of countably many copies of $Z$. In general, Aut $\left(\sigma_{A}\right)$ is a huge noncommutative countable group when $A$ is primitive. Other work on $\operatorname{Aut}\left(\sigma_{A}\right)$ may be found in [BF], [BK1], [BK2], [F], [KRW1], [KRW2], [KRW3], [N], [W1], [W2], [W3], [W4], [W5]. An important normal subgroup of $A u t\left(\sigma_{A}\right)$ is the group $\operatorname{Simp}\left(\sigma_{A}\right)$ of simple automorphisms [N], which arise from automorphisms of graphs that keep the vertices fixed. Consider a directed graph constructed from a matrix $P$ over $Z^{+}$where there are $P_{k l}$ arcs going from the vertex $k$ to the vertex $l$ as in Figure 2 with, for example, $P_{k l}=5$. Any permutation $\alpha$ of the $\operatorname{arcs}$ from $k$ to $l$ induces a simple automorphism $\alpha$ of $\left(X_{P}, \sigma_{P}\right)$. By definition, $\operatorname{Simp}\left(\sigma_{A}\right)$ is generated by automorphisms of $\left(X_{A}, \sigma_{A}\right)$ which are conjugate to those like $\alpha$ for various graphs $P$.

We let $A u t\left(s_{A}\right)$ denote the group of automorphisms of the dimension pair $\left(G_{A}, s_{A}\right)$. By definition, this consists of those automorphisms $\alpha$ of the group $G_{A}$ which commute with the isomorphism $s_{A}$. We do not require $\alpha$ to preserve the nonnegative elements $G_{A}^{+}$. As mentioned in (2.12), the case relevant to this paper is where $\operatorname{det}(A)= \pm 1$. We then have the very concrete description

$$
\operatorname{Aut}\left(s_{A}\right)=\text { the elements } G l_{n}(Z) \text { which commute with } A .
$$

If, in addition, the characteristic polynomial $\operatorname{det}(t I-A)$ is irreducible, we have

$$
Z[A]^{*} \subset A u t\left(s_{A}\right) \subset O^{*} \subset Q[A]^{*}
$$

where $O$ is the ring of integers in the number field $Q[\lambda] \simeq Q[A]$ generated by the Perron-Frobenius eigenvalue $\lambda$ of $A$. The notation $R^{*}$ means the group of invertible elements in a ring $R$. See [LM], [BLR], [BMT]. In particular, using (3.5) it is possible in many cases to explicitly compute generators for $A u t\left(s_{A}\right)$ using the computational algebra program PARI. 
Theorem 3.6. There are isomorphisms

$$
\begin{aligned}
& \pi_{1}(S S E(Z O), A)=\operatorname{Aut}\left(\sigma_{A}\right) \\
& \pi_{i}(S S E(Z O), A)=0 \quad \text { for } i \geq 2 \\
& \pi_{1}\left(S S E\left(Z^{+}\right), A\right)=\operatorname{Aut}\left(\sigma_{A}\right) / \operatorname{Simp}\left(\sigma_{A}\right) \\
& \pi_{1}(S S E(Z), A)=\operatorname{Aut}\left(s_{A}\right) \\
& \pi_{i}(S S E(Z), A)=0 \quad \text { for } i \geq 2 .
\end{aligned}
$$

This is proved in [BaW], [W1], [W2], [W3], [W4].

An important representation of $\operatorname{Aut}\left(\sigma_{A}\right)$ is the dimension group homomorphism

$$
\delta_{A}: \operatorname{Aut}\left(\sigma_{A}\right) \rightarrow \operatorname{Aut}\left(s_{A}\right)
$$

which was first defined dynamically by Kreiger as explained in [BLR], [LM], [W6]. In view of (3.6), it also can be defined using the homomorphism of fundamental groups

$$
\delta_{A}: \pi_{1}(S S E(Z O), A) \rightarrow \pi_{1}\left(S S E\left(Z^{+}\right), A\right) \rightarrow \pi_{1}(S S E(Z), A) .
$$

The image of $\delta_{A}$ actually lies inside the subgroup of automorphisms of $\left(G_{A}, s_{A}\right)$ which preserve $G_{A}^{+}$, although we will not make use of this. If $(R, S): A \rightarrow B$ over $Z O$ or $Z^{+}$, then the induced isomorphism of dimension group triples

$$
g(R):\left(G_{A}, G_{A}^{+}, s_{A}\right) \rightarrow\left(G_{B}, G_{B}^{+}, s_{B}\right)
$$

just comes from the isomorphism from the eventual range $R_{A}$ of $A$ to the eventual range $R_{B}$ of $B$ induced by $R$. Ditto for $(R, S): A \rightarrow B$ over $Z$, except that $G_{A}^{+}$does not necessarily go to $G_{B}^{+}$. Suppose $\gamma$ in $\operatorname{Aut}\left(\sigma_{A}\right), \operatorname{Aut}\left(\sigma_{A}\right) / \operatorname{Simp}\left(\sigma_{A}\right)$, or $A u t\left(s_{A}\right)$ is represented as a loop

$$
\gamma=\prod_{i=1}^{m} \gamma\left(R_{i}, S_{i}\right)^{\epsilon_{i}}
$$

over $Z O, Z^{+}$, or $Z$ respectively where $\gamma\left(R_{i}, S_{i}\right)$ is the path between $A_{i-1}$ and $A_{i}$ corresponding to the strong shift equivalence $\left(R_{i}, S_{i}\right)$ and where $\epsilon_{i}=+1$ if $\left(R_{i}, S_{i}\right): A_{i-1} \rightarrow A_{i}$ and $\epsilon_{i}=-1$ if $\left(R_{i}, S_{i}\right): A_{i} \rightarrow A_{i-1}$. Then using (C) in (3.6) we have

$$
\delta_{A}(\gamma)=\prod_{i=1}^{m} g\left(R_{i}\right)^{\epsilon_{i}} .
$$

Here is how the $S S E$ spaces arose. Recall the following version of Williams' Theorem in the category $Z O$.

Theorem 3.11. Let $\gamma:\left\{X_{A}, \sigma_{A}\right\} \rightarrow\left\{X_{B}, \sigma_{B}\right\}$ be a topological conjugacy where $A$ and $B$ are in $Z O$. Then there is a chain of elementary strong shift equivalences $\left(R_{1}, S_{1}\right), \ldots,\left(R_{m}, S_{m}\right)$ between $A$ and $B$ in $Z O$ such that

$$
\gamma=\prod_{i=1}^{m} c\left(R_{i}, S_{i}\right)^{\epsilon_{i}}
$$

where $\epsilon_{i}=+1$ if $\left(R_{i}, S_{i}\right): A_{i-1} \rightarrow A_{i}$ and $\epsilon_{i}=-1$ if $\left(R_{i}, S_{i}\right): A_{i} \rightarrow A_{i-1}$.

There are many ways in which $\gamma$ can be written as such a product corresponding to different "paths" of elementary strong shift equivalences connecting $A$ and $B$. It is natural to ask if there are some general relations between the various $c(R, S)$ which would correspond to deformations or homotopies between paths connecting $A$ 
and $B$. Put slightly differently, is there a natural notion of deformation or homotopy between paths connecting $A$ and $B$ such that all homotopic paths give the same $\gamma$ ? And does this notion of homotopy capture all relations between the $c(R, S)$ ? The key to answering these questions is to consider the set of topological Markov partitions to be like the contractible space of real valued functions on a manifold. In fact, the set $P_{A}$ of all topological Markov partitions of a subshift of finite type $\left\{X_{A}, \sigma_{A}\right\}$ can be given the structure of a simplicial complex, as explained below, which turns out to be contractible [BaW], [W1]. The main point in Williams' proof of his classification theorem really amounts to showing that $P_{A}$ is connected. See $[\mathrm{Fr}],[\mathrm{W} 1],[\mathrm{Wi1}]$.

Definition 3.12. See [PT, W1]. Let $f: X \rightarrow X$ be a homeomorphism of a zerodimensional compact space $X$. A topological Markov partition for $(X, f)$ is a finite covering $U=\left\{U_{i}\right\}$ of $X$ such that

(MP1) The sets $U_{i}$ are nonempty, open, and disjoint.

(MP2) Any intersection $\bigcap_{-\infty<n<\infty} f^{-n}\left(U_{i(n)}\right)$ consists of at most one point.

(MP3) If $U_{i(n)} \cap f^{-1}\left(U_{i(n+1)}\right)$ is not empty for all $n$, then the intersection $\bigcap_{-\infty<n<\infty} f^{-n}\left(U_{i(n)}\right)$ is not empty.

Note. The sets in $U$ are not listed in any particular order.

The standard Markov partition $U^{A}=\left\{U_{i}^{A}\right\}$ for a subshift of finite type $\left\{X_{A}, \sigma_{A}\right\}$ of $\left(X_{m}, \sigma_{m}\right)$ is obtained by letting $U_{i}^{A}=\left\{x \epsilon X_{A} \mid x_{0}=i\right\}$.

Here is an outline of the proof for (3.11). If $U=\left\{U_{i}\right\}$ and $V=\left\{V_{j}\right\}$ are partitions (not necessarily Markov) of the same set $X$, let $U \cap V=\left\{U_{i} \cap V_{j}\right\}$ where any empty intersection $U_{i} \cap V_{j}$ is not counted. Let $U<V$ mean that $V$ refines $U$; i.e., each set $V_{j}$ is contained in some $U_{i}$. If $f: X \rightarrow Y$ is a function and $V=\left\{V_{j}\right\}$ is a partition of $Y$, let $f^{-1}(V)=\left\{f^{-1}\left(V_{j}\right)\right\}$. Now suppose $U=\left\{U_{i}\right\}$ and $V=\left\{V_{j}\right\}$ are topological Markov partitions of $\left\{X_{A}, \sigma_{A}\right\}$. As in [W1] we write

$$
U \rightarrow V
$$

to mean that $U<U \cap V<U \cap \sigma_{A}^{-1}(U)$ and $V<U \cap V<\sigma_{A}(V) \cap V$. At this point we

$$
\text { choose an ordering for the sets } U_{i} \text { in each topological }
$$
Markov partition $U$.

Define matrices $R=R(U, V)=\left\{R_{i j}\right\}$ and $S=S(V, U)=\left\{S_{j i}\right\}$ by the conditions

$$
\begin{array}{ll}
R_{i j}=1 & \text { iff } U_{i} \cap V_{j} \text { is not empty } \\
S_{j i}=1 & \text { iff } V_{j} \cap \sigma_{A}^{-1}\left(U_{i}\right) \text { is not empty }
\end{array}
$$

and verify that

$$
M(U)=R(U, V) S(V, U) \quad M(V)=S(V, U) R(U, V) .
$$

Now let $\gamma:\left\{X_{A}, \sigma_{A}\right\} \rightarrow\left\{X_{B}, \sigma_{B}\right\}$ be a topological conjugacy as in (3.11). Let $V=\gamma^{-1}\left(U^{B}\right)$. This is a topological Markov partition of $\left\{X_{A}, \sigma_{A}\right\}$. Let $C=M(V)$. The orderings chosen in (3.14) together with the bijection between the sets of $V$ and the sets of $U^{B}$ given by $\gamma$ determine a permutation matrix $P$ such that there is an elementary strong shift equivalence $\left(P, P^{-1} C\right): C \rightarrow B$. Williams' argument [Wi1], [Fr], [W1] shows there is a path of "edges" of the form $U \rightarrow V$ connecting $U^{A}$ to $V$. Apply (3.16) to each edge in this path to get a sequence of elementary strong shift equivalences over $Z O$ connecting $A=M\left(U^{A}\right)$ to $C=M(V)$. Then one 
shows $\gamma$ is the product of the corresponding conjugacies $c(R, S)^{ \pm 1}$ together with $c\left(P, P^{-1} C\right)$.

We start to build the space of Markov partitions $P_{A}$ by letting the vertices be Markov partitions of $\left\{X_{A}, \sigma_{A}\right\}$. The edges are ordered pairs $[U, V]$ where $U \rightarrow V$. Different product expressions in (3.11) arise from choosing different paths from $U^{A}$ to $V$ in the proof above. So what is the right definition of homotopy or deformation of paths in $P_{A}$ ? Consider an ordered triple $[U, V, W]$ where

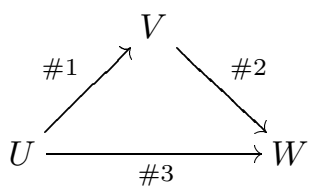

For $i=1,2,3$, define $\left(R_{i}, S_{i}\right)$ using (3.15) on the corresponding edge in (3.17). Then $R_{1} R_{2}=R_{3}$, and the following was proved in [W2].

Triangle Lemma 3.18. (A) The three $\left(R_{i}, S_{i}\right)$ coming from (3.17) satisfy the Triangle Identities. (B) If we have any diagram like (3.1) over $Z O$ and the Triangle Identities hold, then

$$
c\left(R_{3}, S_{3}\right)=c\left(R_{1}, S_{1}\right) c\left(R_{2}, S_{2}\right) .
$$

(C) Suppose we have a diagram like (3.1) over $Z O$ for which $R_{1} R_{2}=R_{3}$, and suppose the composition rule in $(B)$ holds. Then the last two equations in the Triangle Identities must hold.

Part (C) says the last two equations of the Triangle Identities are forced by the very natural composition law for the $c\left(R_{i}, S_{i}\right)$ in the presence of the equation $R_{1} R_{2}=R_{3}$ ! The simplicial structure on $P_{A}$ given in [W1] defines an $n$-simplex to be an ordered n-tuple $\left[U^{0}, U^{1}, \ldots, U^{n}\right]$ of topological Markov partitions of $\left\{X_{A}, \sigma_{A}\right\}$ such that $U^{i} \rightarrow U^{j}$ whenever $i<j$. The Triangle Lemma justified the definition for the 2-cells of $S S E(Z O)$, and it suggested defining the higher n-cells as in (9.5) below. The result was (A) of Theorem 3.6. This was very encouraging from the viewpoint of topology, because it said that $S S E(Z O)$ is a construction of the classifying space of the discrete group $A u t\left(\sigma_{A}\right)$ which is quite different from the universal bar construction method in homological algebra. Such circumstances have historically tended to yield new information.

\section{The $\Delta$-STRATEgY}

The exact homotopy sequence of the pair $\left(S S E(\Lambda), S S E\left(\Lambda^{+}\right)\right)$leads to a strategy for producing counterexamples to the Strong Shift Equivalence Problem which was observed independently by Kim-Roush and by the author.

Let $\Lambda$ be a ring containing 1 and with a set of nonnegative elements $\Lambda^{+}$which contains 0 and 1 and which is closed under addition and multiplication. We will assume $\Lambda^{+}$satisfies the condition that if $a$ and $b$ are in $\Lambda^{+}$and $a+b=0$ or $a b=0$, then either $a=0$ or $b=0$. Typical examples of $\Lambda$ are the integers $Z$, a subring of the real numbers, a ring of polynomials in commuting or noncommuting variables with integer coefficients, a ring of Laurent polynomials with integer coefficients, and the integral group ring $Z[G]$ of a group $G$.

Let $S^{+}$denote a union of components in $S S E\left(\Lambda^{+}\right)$. For example $S^{+}$could be $S S E_{m}\left(\Lambda^{+}\right)$, which consists of those components of $S S E\left(\Lambda^{+}\right)$containing vertices $A$ 
satisfying

$$
\operatorname{Trace}(A)=\cdots=\operatorname{Trace}\left(A^{m}\right)=0 .
$$

Let $\pi_{1}\left(S S E(\Lambda), S^{+}\right)$denote homotopy classes of paths in $S S E(\Lambda)$ with endpoints in $S^{+}$. We would like to find a function

$$
\Delta: \pi_{1}\left(\operatorname{SSE}(\Lambda), S^{+}\right) \rightarrow G
$$

where $G$ is, say, an abelian group such that $\Delta$ satisfies the properties

$$
\begin{aligned}
& \Delta(\alpha * \beta)=\Delta(\alpha)+\Delta(\beta), \\
& \Delta(\alpha)=0 \text { whenever } \alpha \text { lies in } S^{+} .
\end{aligned}
$$

Let $A$ and $B$ be vertices in $S^{+}$, and choose a path $\alpha$ from $A$ to $B$ in $S S E(\Lambda)$. If $\beta$ is another path from $A$ to $B$, we have

$$
\Delta(\alpha)=\Delta(\beta)+\Delta\left(\alpha * \beta^{-1}\right) .
$$

Consequently, there is an invariant

$$
\Delta(A, B)=\Delta(\beta) \quad \text { in } \quad G \bmod \quad \Delta\left(\pi_{1}(S S E(\Lambda), A)\right)
$$

which vanishes if there is a path from $A$ to $B$ in $S^{+}$. In particular, a counterexample to the Strong Shift Equivalence Problem can be obtained by finding a function $\Delta$ together with matrices $A$ and $B$ such that

$$
\begin{aligned}
& \Delta(A, B)=\Delta(\beta) \neq 0 \text { for some } \beta, \\
& \Delta(\alpha)=0 \text { whenever } \alpha \text { is in } \pi_{1}(\operatorname{SSE}(\Lambda), A) .
\end{aligned}
$$

\section{A counterexample to the Strong Shift Equivalence Problem for} PRIMITIVE MATRICES

In this section we give an example of the $\Delta$-strategy for $\Delta=s g c_{2}=\Phi_{2}$. In Section 6 and Section 7 we discuss how to construct $s g c_{k}$ and $\Phi_{2 m}$ respectively. The method for finding candidate matrices $A$ and $B$ comes from [KR5], [KR6]. Let $M$ be the $4 \times 4$ matrix

$$
\left(\begin{array}{llll}
0 & 0 & 1 & 1 \\
1 & 0 & 0 & 0 \\
0 & 1 & 0 & 0 \\
0 & 0 & 1 & 0
\end{array}\right)
$$

from [KRW1, 4.1] with characteristic polynomial $t^{4}-t-1$. It satisfies the equation

$$
(M-I)\left(M^{4}+M^{3}+M^{2}\right)=M .
$$

Let $\alpha$ be the loop $(E, F): M \rightarrow M$ where

$$
E=M-I=\left(\begin{array}{llll}
-1 & 0 & 1 & 1 \\
1 & -1 & 0 & 0 \\
0 & 1 & -1 & 0 \\
0 & 0 & 1 & -1
\end{array}\right) \quad F=M^{4}+M^{3}+M^{2}=\left(\begin{array}{llll}
2 & 2 & 2 & 1 \\
1 & 2 & 2 & 1 \\
1 & 1 & 2 & 1 \\
1 & 1 & 1 & 1
\end{array}\right) .
$$

Direct computation using (6.1) below shows $\Delta(\alpha) \neq 0$. Next we want to extend $E$ and $F$ to matrices of the form

$$
R=\left(\begin{array}{cc}
E & 0 \\
0 & I
\end{array}\right) \quad S=\left(\begin{array}{cc}
F & X \\
Y & Z
\end{array}\right)
$$

so that letting $A=R S, B=S R$, and $\beta$ be the path $(R, S): A \rightarrow B$, we have 
(I) $A$ and $B$ are primitive, nonnegative integral matrices with

$\operatorname{det}(A)=\operatorname{det}(B)= \pm 1$ and

$\operatorname{Trace}(A)=\operatorname{Trace}(B)=\operatorname{Trace}\left(A^{2}\right)=\operatorname{Trace}\left(B^{2}\right)=0$.

(II) $\Delta(\beta) \neq 0$.

(III) $\Delta(\alpha)=0$ for all $\alpha$ in $\pi_{1}(S S E(Z), A) \simeq \operatorname{Aut}\left(s_{A}\right)$.

Trial and error and luck produce

$$
X=\left(\begin{array}{lll}
2 & 0 & 0 \\
2 & 0 & 0 \\
2 & 0 & 0 \\
2 & 0 & 0
\end{array}\right) \quad Y=\left(\begin{array}{llll}
0 & 0 & 0 & 0 \\
1 & 1 & 2 & 1 \\
1 & 1 & 2 & 1
\end{array}\right) \quad Z=\left(\begin{array}{lll}
0 & 0 & 1 \\
3 & 0 & 0 \\
0 & 1 & 0
\end{array}\right)
$$

and therefore

$$
A=R S=\left(\begin{array}{ccccccc}
0 & 0 & 1 & 1 & 2 & 0 & 0 \\
1 & 0 & 0 & 0 & 0 & 0 & 0 \\
0 & 1 & 0 & 0 & 0 & 0 & 0 \\
0 & 0 & 1 & 0 & 0 & 0 & 0 \\
0 & 0 & 0 & 0 & 0 & 0 & 1 \\
1 & 1 & 2 & 1 & 3 & 0 & 0 \\
1 & 1 & 2 & 1 & 0 & 1 & 0
\end{array}\right) \quad B=S R=\left(\begin{array}{ccccccc}
0 & 0 & 1 & 1 & 2 & 0 & 0 \\
1 & 0 & 0 & 0 & 2 & 0 & 0 \\
0 & 1 & 0 & 0 & 2 & 0 & 0 \\
0 & 0 & 1 & 0 & 2 & 0 & 0 \\
0 & 0 & 0 & 0 & 0 & 0 & 1 \\
0 & 1 & 0 & 0 & 3 & 0 & 0 \\
0 & 1 & 0 & 0 & 0 & 1 & 0
\end{array}\right) .
$$

Direct computation shows Property I holds. Since $\Delta(\alpha) \neq 0$, the formula (6.1) shows that Property II holds for any $X$ and $Y$ and any $Z$ with diagonal entries zero because $E$ was extended by adding on the identity matrix. To verify Property III, first compute the characteristic polynomial for $A$. It is $t^{7}-6 t^{4}-5 t^{3}-6 t^{2}-37+1$. Putting this into the command "buchgenfu()" of PARI gives

$$
Z[A]^{*}=\operatorname{Aut}\left(s_{A}\right)=O^{*}=Z[\lambda]^{*}
$$

in (3.5) because $\left\{1, \lambda, \lambda^{2}, \ldots, \lambda^{6}\right\}$ is a basis for $O$. The Dirichlet Unit Theorem shows the rank of $O^{*}$ is 4 , and PARI computes a set of generators for $O^{*}$ to be

$$
\begin{aligned}
& R_{0}=-1 \\
& R_{1}=\lambda \\
& R_{2}=2 \lambda^{6}-\lambda^{5}+\lambda^{4}-13 \lambda^{3}-4 \lambda^{2}-13 \lambda-1 \\
& R_{3}=\lambda^{6}+\lambda^{4}-7 \lambda^{3}-6 \lambda^{2}-8 \lambda-6 \\
& R_{4}=2 \lambda^{6}-4 \lambda^{5}-2 \lambda^{3}+3 \lambda .
\end{aligned}
$$

Substitute $A$ for $\lambda$ in these expressions for each $i=0,1,2,3,4$ to get five loops $\alpha_{i}=$ $\left(R_{i}, R_{i}^{-1} A\right): A \rightarrow A$ generating $\pi_{1}(S S E(Z), A)=A u t\left(s_{A}\right) . \Delta\left(\alpha_{0}\right)=0$ because $R_{0}$ is diagonal and the diagonal entries of $A$ are zero. Computer computation using the explicit formula (6.1) shows $\Delta\left(\alpha_{i}\right)=0$ for each $i=1,2,3,4$.

\section{ThE SIGN-GYRATION-COMPATIBILITY-CONDITION}

Consider an edge $(R, S): M \rightarrow N$ in $S S E(Z)$. Let

$$
\operatorname{sgc}_{2}(R, S)=\sum_{i<j, k>l} R_{i k} S_{k i} R_{j l} S_{l j}+\sum_{i<j, k \geq l} R_{i k} S_{k j} R_{j l} S_{l i}+\sum_{i, k} \frac{R_{i k}\left(R_{i k}-1\right)}{2} S_{k i}^{2}
$$

in $Z / 2 Z$. Let $A$ and $B$ be vertices in $S S E_{2}\left(Z^{+}\right)$. Consider a path $\gamma$ from $A$ to $B$ in $S S E(Z)$. Write $\gamma$ as a concatenation

$$
\gamma=\prod_{k=1}^{m} \gamma\left(R_{k}, S_{k}\right)^{\epsilon_{k}}
$$


where $\epsilon_{k}=+1$ if $\left(R_{k}, S_{k}\right): A_{k-1} \rightarrow A_{k}$ and $\epsilon_{k}=-1$ if $\left(R_{k}, S_{k}\right): A_{k} \rightarrow A_{k-1}$.

Theorem 6.3. The formula

$$
\operatorname{sgc}_{2}(\gamma)=\sum_{k=1}^{m} \epsilon_{k} s g c_{2}\left(R_{k}, S_{k}\right) \quad \bmod 2
$$

defines a function

$$
\operatorname{sgc}_{2}: \pi_{1}\left(S S E(Z), S S E_{2}\left(Z^{+}\right)\right) \rightarrow Z / 2 Z
$$

satisfying (4.2).

The rather complicated formula (6.1) and Theorem 6.3 have a history going back over ten years. The conceptual background comes from studying $\operatorname{Aut}\left(\sigma_{A}\right)$. Two important representations of $A u t\left(\sigma_{A}\right)$ are the dimension group representation $\delta_{A}$ discussed above and the sign and gyration number homomorphisms discussed below. The first is essentially a matrix group representation, and the second take values in finite cyclic groups. The link between them is provided by the signgyration-compatibility-condition relations, and a prominent role is played by the subgroup

$$
\operatorname{Inert}\left(\sigma_{A}\right)=\operatorname{Kernel}\left(\delta_{A}\right)
$$

of inert automorphisms.

In [BK1] Boyle and Krieger defined the sign and gyration number homomorphisms

$$
\begin{array}{cc}
O S_{m}: \operatorname{Aut}\left(\sigma_{A}\right) \rightarrow Z / 2 & \text { for } m \geq 1 \\
G Y_{m}: \operatorname{Aut}\left(\sigma_{A}\right) \rightarrow Z / m & \text { for } m \geq 2 .
\end{array}
$$

$O S_{m}(\alpha)$ is the sign of the permutation $\alpha$ induces on the orbits of length $m$, and $G Y_{m}(\alpha)$ is the average measure of how $\alpha$ moves orbits of length $m$ parallel to themselves. To define $G Y_{m}(\alpha)$, list the orbits of length $m$ and choose a point $b_{i}$ on the $\mathrm{i}^{\text {th }}$ orbit. Write $\sigma_{A}\left(b_{i}\right)=\sigma_{A}^{r_{i}}\left(b_{j}\right)$. Then

$$
G Y_{m}(\alpha)=\sum_{i} r_{i} \quad \bmod \quad m .
$$

This is independent of the choice of base points $b_{i}$. Boyle and Krieger [BK1] proved that certain sign-gration-compatibility-condition relations hold between $G Y_{m}(\alpha)$ and various $O S_{m / 2^{r}}(\alpha)$ for involutions $\alpha$ in $A u t\left(\sigma_{n}\right)$. This was subsequently proved for simple automorphisms by Nasu $[\mathrm{N}]$, for finite order inert automorphisms by Fiebig $[\mathrm{F}]$, and finally for all inert automorphisms in [KR3], [KRW1]. These results may be interpreted as vanishing of the so-called sign-gyration-compatibilitycondition homomorphism

$$
S G C C_{k}: \operatorname{Aut}\left(\sigma_{A}\right) \rightarrow Z / k Z
$$

on various types of elements of $\operatorname{Aut}\left(\sigma_{A}\right)$. See [KRW1]. By definition

$$
S G C C_{k}=G Y_{k}+\sum_{i>0} O S_{k / 2^{i}}
$$

where $O S_{k / 2^{i}}=0$ if $k / 2^{i}$ is not integral and where $Z / 2 Z$ is identified with the subgroup $\{0, k / 2\}$ of $Z / k Z$ when $k$ is even. For example, when $k=2$ we have

$$
S G C C_{2}=G Y_{2}+O S_{1} \text {. }
$$


The expression (6.1) is simply the explicit algebraic formula [KRW1] for the dynamically defined $S G C C_{2}$ on the conjugacy $c(R, S):\left(X_{A}, \sigma_{A}\right) \rightarrow\left(X_{B}, \sigma_{B}\right)$ arising from an elementary strong shift equivalence $(R, S): A \rightarrow B$ over $Z^{+}$computed with respect to the lexicographical ordering of fixed points and points of period two. In [KRW1] it was shown that there is a commutative diagram

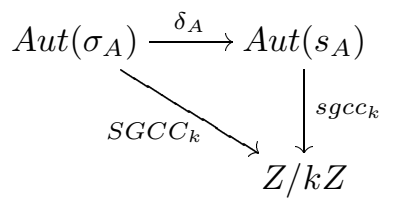

when $A$ is primitive. The setting for the proof of (6.5) and (6.3) is (3.8). It was the construction of $s g c c_{k}$ which led directly to the $\Delta$-strategy and to the proof (6.3) in the primitive case.

The main point is that the algebraic expression (6.1) makes sense for any $(R, S)$ : $A \rightarrow B$ over $Z$, not just over $Z^{+}$, and that homotopy invariance is satisfied. Namely,

Proposition 6.6. The cocyle condition

$$
s g c_{2}\left(R_{3}, S_{3}\right)=\operatorname{sgc}_{2}\left(R_{1}, S_{1}\right)+s g c_{2}\left(R_{2}, S_{2}\right)
$$

holds for a triangle in $S S E(Z)$.

This was first verified in [KRW1] under the condition that the vertices in the triangle lie in a component of $S S E(Z)$ which contains a primitive nonnegative integral matrix. Mike Boyle subsequently found a proof, given in [KR6], which eliminates the primitivity condition and vastly simplifies the presentation in [KRW1].

Here is Boyle's argument. It is related to the cyclic structure on the $S S E$ spaces described in Number 8 of Section 9. First suppose the triangle (3.1) actually lies in $S S E\left(Z^{+}\right)$. In [BK1] and [KRW1] it was shown that the cocycle condition holds because $s g c_{2}$ is simply the algebraic formula for the dynamically defined homomorphism $S G C C_{2}$. Now suppose (3.1) lies in $S S E(Z)$. If $M=\{M(i, j)\}$ is a matrix over $Z$, let $|M|=\sum_{i j}|M(i, j)|$. Define nonnegative matrices $R_{1}^{\prime}, R_{2}^{\prime}$, and $S_{3}^{\prime}$ by the equations

$$
\begin{aligned}
& R_{1}^{\prime}(i, j)=R_{1}(i, j)+4\left|R_{1}\right| \\
& R_{2}^{\prime}(j, k)=R_{2}(j, k)+4\left|R_{2}\right| \\
& S_{3}^{\prime}(k, i)=S_{3}(k, i)+4\left|S_{3}\right| .
\end{aligned}
$$

Let $R_{3}^{\prime}=R_{1}^{\prime} R_{2}^{\prime}, S_{1}^{\prime}=R_{2}^{\prime} S_{3}^{\prime}$, and $S_{2}^{\prime}=S_{3}^{\prime} R_{1}^{\prime}$. Let $A^{\prime}=R_{1}^{\prime} S_{1}^{\prime}, B^{\prime}=R_{2}^{\prime} S_{2}^{\prime}$, and $C^{\prime}=S_{3}^{\prime} R_{3}^{\prime}$. We then get a triangle

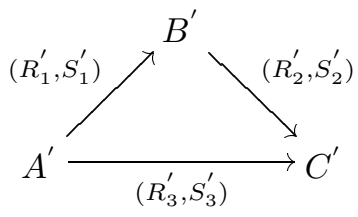

in $\operatorname{SSE}\left(Z^{+}\right)$and $s g c_{2}\left(R_{p}, S_{p}\right)=\operatorname{sgc}_{2}\left(R_{p}^{\prime}, S_{p}^{\prime}\right)$ modulo 2 for $p=1,2,3$. 
This argument generalizes essentially verbatim with $k(2 k)$ ! replacing the coefficient 4 to produce cohomology classes

$$
s g c_{k}: \pi_{1}\left(S S E(Z), S S E_{k}\left(Z^{+}\right)\right) \rightarrow Z / k Z
$$

satisfying (4.2), although the polynomial expressions for $S G C C_{k}(R, S)$, and hence for $s g c_{k}$, become complicated as $k$ grows. In the primitive case $s g c_{k}=s g c c_{k}$. See [KR6].

\section{An SSE inVARIant in $K_{2}$ OF the DUAL NUmbers}

Two invertible matrices $X$ and $Y$ over a ring $R$ determine the same element in the algebraic K-theory group $K_{1}(R)$ iff $E X F=Y$ where $E$ and $F$ are products of elementary matrices $e_{i j}(r)$. By definition, $e_{i j}(r)$ is the invertible matrix which is the identity $I$ on the diagonal, has the element $r$ of $R$ in the $\mathrm{i}^{\text {th }}$ row and $\mathrm{j}^{\text {th }}$ column where $i \neq j$, and is zero elsewhere. Roughly speaking, the abelian group $K_{2}(R)$ is a measure of the different ways to get from $X$ to $Y$ using such row and column operations. In particular, any product of elementary matrices

$$
e_{i_{1} j_{1}}\left(r_{1}\right) e_{i_{2} j_{2}}\left(r_{2}\right) \cdots e_{i_{p} j_{p}}\left(r_{p}\right)=I
$$

gives rise to an element of $K_{2}(R)$. See [Mi], [R].

Here are the precise definitions of $K_{1}(R)$ and $K_{2}(R)$. Let $E(R)$ denote the subgroup of the general linear group $G l(R)$ generated by the elementary matrices $e_{i j}(r)$. The Whitehead Lemma says that $E(R)$ is the commutator subgroup of $G l(R)$. Define the abelian group $K_{1}(R)$ by the equation

$$
K_{1}(R)=G l(R) /[G l(R), G l(R)]=G l(R) / E(R) .
$$

If $R$ is a commutative ring, then the determinant function $\operatorname{det}(A)$ of a matrix $A$ defines a homomorphism

$$
\text { det }: K_{1}(R) \rightarrow R^{*}
$$

which is actually an isomorphism for many rings. This is the case, for example, if $R$ is a principal ideal domain or the ring of integers in a number field. To obtain $K_{2}$, first define the Steinberg group $S t(R)$ by generators $x_{i j}(r)$ and relations

(ST1) $x_{i j}(r+s)=x_{i j}(r) x_{i j}(s)$

(ST2) $\left[x_{i j}(r), x_{k l}(s)\right]=1$ if $i \neq l$ and $j \neq k$

(ST3) $\left[x_{i j}(r), x_{j k}(s)\right]=x_{i k}(r s)$ if $i, j, k$ are distinct.

These relations are satisfied by the corresponding matrices $e_{i j}(r)$, and so the function $\phi\left(x_{i j}(r)\right)=e_{i j}(r)$ defines a homomorphism

$$
\phi: S t(R) \rightarrow E(R) .
$$

By definition

$$
K_{2}(R)=\text { Kernel of } \phi .
$$

The resulting exact sequence

$$
0 \rightarrow K_{2}(R) \rightarrow S t(R) \rightarrow E(R) \rightarrow 1
$$

is the universal central extension of $E(R)$. This definition of $K_{2}$, due to John Milnor, is related to Robert Steinberg's work on universal covering groups of algebraic groups. $K_{2}$ is also closely connected to reciprocity laws in algebraic number theory. 
Consider the truncated polynomial ring $\Lambda[t] /\left(t^{m+1}\right)$, which is called the dual numbers when $m=1$. Let $K_{2}\left(\Lambda[t] /\left(t^{m+1}\right),(t)\right)$ be the cokernel of the split injection $K_{2}(\Lambda) \rightarrow K_{2}\left(\Lambda[t] /\left(t^{m+1}\right)\right)$. For example, van der Kallen $[\mathrm{vdK}]$ showed that

$$
K_{2}\left(Z[t] /\left(t^{2}\right),(t)\right) \simeq Z / 2 Z
$$

and

$$
K_{2}\left(\Lambda[t] /\left(t^{2}\right),(t)\right) \simeq \Omega_{\Lambda / Z}^{1}
$$

if $\Lambda$ is a commutative ring containing $1 / 2$. Further information on these relative K-groups may be found in [vdK], [Bl], [Ke], [Lo], [MS], [St].

Theorem 7.3. The procedure in Step 1, Step 2, and Step 3 below defines a function

$$
\Phi_{2 m}: \pi_{1}\left(S S E(\Lambda), S S E_{2 m}\left(\Lambda^{+}\right)\right) \rightarrow K_{2}\left(\Lambda[t] /\left(t^{m+1}\right),(t)\right)
$$

satisfying (4.2). Moreover, assume $\Lambda=Z$ and consider a path $(R, S): A \rightarrow B$ in $S S E(Z)$ where $A$ and $B$ are nonnegative matrices with $\operatorname{Trace}(A)=\operatorname{Trace}\left(A^{2}\right)=$ $\operatorname{Trace}(B)=\operatorname{Trace}\left(B^{2}\right)=0$. Then $\Phi_{2}(R, S)=\operatorname{sgc}_{2}(R, S)$ in $Z / 2 Z$.

The function $\Phi_{2 m}$ was constructed in [W7] where an algorithm for evaluating $\Phi_{2}(R, S)$ when $\Lambda=Z$ was given using van der Kallen's paper [vdK]. Machine computations of $\Phi_{2}$ also detected the counterexamples to the Strong Shift Equivalence Problem given in Section 5 and [KR6]. Further numerical evidence raised the question whether $\Phi_{2}=s g c_{2}$, and this was subsequently shown to be true by Kim-Roush in the Appendix to [W7].

The machinery of algebraic K-theory may be useful in computing the indeterminacy which occurs in (4.3) and (4.4) in the strategy for finding counterexamples to the Strong Shift Equivalence Problem. Assume $\Lambda$ is a commutative ring, so that $\operatorname{det}(I-t A)$ makes sense. It was shown in [W7] that $\operatorname{det}(I-t A)=1 \bmod t^{m+1}$ whenever $\operatorname{Tr}(A)=\ldots=\operatorname{Tr}\left(A^{m}\right)=0$ and $A \geq 0$ and that the image of $\Phi_{2 m}$ on $\pi_{1}(S S E(\Lambda), A)$ is contained in the image of the resulting homomorphism

$$
K_{2}\left(\Lambda[t]\left[\frac{1}{\operatorname{det}(I-t A)}\right]\right) \rightarrow K_{2}\left(\Lambda[t] /\left(t^{m+1}\right)\right) \rightarrow K_{2}\left(\Lambda[t] /\left(t^{m+1}\right),(t)\right) .
$$

If $\Lambda$ is a Noetherian ring, there is the localization exact sequence

$$
\ldots K_{2}(\Lambda[t]) \rightarrow K_{2}\left(\Lambda[t]\left[\frac{1}{\operatorname{det}(I-t A)}\right]\right) \rightarrow K_{1}(\Lambda[t] /(\operatorname{det}(I-t A))) \rightarrow K_{1}(\Lambda[t]) \ldots
$$

See [Lo, p. 357] or [R, p. 294]. If $\Lambda$ is also regular [R, p. 110], then $K_{n}(\Lambda[t])=$ $K_{n}(\Lambda)$ for all $n$ [R, p. 295], the localization sequence is split exact, and we have isomorphisms

$$
K_{2}\left(\Lambda[t]\left[\frac{1}{\operatorname{det}(I-t A)}\right]\right) / K_{2}(\Lambda) \simeq K_{1}(\Lambda[t] /(\operatorname{det}(I-t A))) .
$$

This discussion gives an "explanation" of what is happening when we show that $s g c_{2}=\Phi_{2}=0$ on $\pi_{1}(S S E(Z), A)=A u t\left(s_{A}\right)=Z[A]^{*}=K_{1}(Z[A])$ for the particular $A$ appearing in Section 5. It also shows that computing generators for $K_{1}(\Lambda[t] /(\operatorname{det}(I-t A)))$ is key for examining examples over other rings $\Lambda$. As mentioned above, the case $\Lambda=Z\left[t_{1}, t_{1}^{-1}, \ldots, t_{n}, t_{n}^{-1}\right]$ comes up in studying Markov chains [MT1], [MT2], [MT3], [T].

Here is an outline of the construction of $\Phi_{2}$. On the one hand, there are the strong shift equivalence equations and the strong shift equivalence spaces of Section 3. On the other hand, the algebraic K-theory groups $K_{1}$ and $K_{2}$ arise from row 
and column operations on matrices. The basic idea is to use the polynomial strong shift equations of [BW], [W7] which convert an elementary strong shift equivalence $(P, Q): M \rightarrow N$ into a sequence of row and column matrix operations leading from $I-t M$ to $I-t N$. This is analogous to one-parameter families of functions which pseudo-isotopy theory $[\mathrm{C}],[\mathrm{HW}]$ relates to $K_{2}$.

PSSE Equations

$$
\begin{aligned}
\left(\begin{array}{cc}
I-t P Q & 0 \\
-t Q & I
\end{array}\right)\left(\begin{array}{cc}
I & 0 \\
t Q & I
\end{array}\right) & =\left(\begin{array}{cc}
I-t P Q & 0 \\
0 & I
\end{array}\right) \\
\left(\begin{array}{cc}
I & P \\
0 & I
\end{array}\right)\left(\begin{array}{cc}
I & -P \\
-t Q & I
\end{array}\right) & =\left(\begin{array}{cc}
I-t P Q & 0 \\
-t Q & I
\end{array}\right) \\
\left(\begin{array}{cc}
I & -P \\
-t Q & I
\end{array}\right)\left(\begin{array}{cc}
I & P \\
0 & I
\end{array}\right) & =\left(\begin{array}{cc}
I & 0 \\
-t Q & I-t Q P
\end{array}\right) \\
\left(\begin{array}{cc}
I & 0 \\
t Q & I
\end{array}\right)\left(\begin{array}{cc}
I & 0 \\
-t Q & I-t Q P
\end{array}\right) & =\left(\begin{array}{cc}
I & 0 \\
0 & I-t Q P
\end{array}\right)
\end{aligned}
$$

The strong shift equivalence equations led to the polynomial strong shift equations in the early 1980's, and this suggested that it might be fruitful to explore analogies between algebraic K-theory and symbolic dynamics. The result was the SSE spaces and $s g c_{k}$ cohomology classes. Then in the last few years the polynomial matrix viewpoint reappeared and has been used in [BW], [KRW2], [KRW3], [KOR], [W7].

Step 1. Let $(P, Q): M \rightarrow N$ be a strong shift equivalence over $\Lambda$. Consider the products of elementary matrices

$$
\begin{aligned}
& W_{L}(P, Q)=\left(\begin{array}{cc}
I & 0 \\
t Q & I
\end{array}\right)\left(\begin{array}{cc}
I & -P \\
0 & I
\end{array}\right) \\
& W_{R}(P, Q)=\left(\begin{array}{cc}
I & 0 \\
-t Q & I
\end{array}\right)\left(\begin{array}{cc}
I & P \\
0 & I
\end{array}\right) \\
& E_{L}(P, Q)=W_{L}(I, N)^{-1} W_{L}(P, Q) \\
& E_{R}(P, Q)=W_{R}(P, Q) W_{R}(I, N)^{-1} .
\end{aligned}
$$

We have the matrix equation

$$
E_{L}(P, Q)\left(\begin{array}{cc}
I-t M & 0 \\
0 & I
\end{array}\right) E_{R}(P, Q)=\left(\begin{array}{cc}
I-t N & 0 \\
0 & I
\end{array}\right) .
$$

Step 2. Let $M$ be a matrix over $\Lambda^{+}$such that $\operatorname{Tr}(M)=0$. Then each diagonal entry $M_{i i}$ is zero. Consider the products of elementary matrices

$$
\begin{aligned}
L_{M} & =\prod_{j=1}^{s-1} \prod_{i=j+1}^{s} e_{i j}\left(t M_{i j}\right) \\
R_{M} & =\prod_{i=1}^{s-1} \prod_{j=i+1}^{s} e_{i j}\left(t M_{i j}\right) .
\end{aligned}
$$

We have the matrix equation

$$
L_{M}(I-t M) R_{M}=I-t^{2} M^{\prime}
$$


for some matrix $M^{\prime}$ over the polynomial ring $\Lambda[t]$.

Step 3. Let $A$ and $B$ be matrices over $\Lambda^{+}$with $\operatorname{Tr}(A)=\operatorname{Tr}(B)=0$, and let $\gamma$ be a path in $S S E(\Lambda)$ connecting $A$ and $B$. Apply Step 1 to each $\operatorname{arc}(P, Q): M \rightarrow N$ in $\gamma$ to get products of elementary matrices $E_{L}(\gamma)$ and $E_{R}(\gamma)$ satisfying the matrix equation

$$
E_{L}(\gamma)(I-t A) E_{R}(\gamma)=I-t B
$$

Step 2 yields

$$
L_{B} E_{L}(\gamma) L_{A}^{-1}\left(I-t^{2} A^{\prime}\right) R_{A}^{-1} E_{R}(\gamma) R_{B}=I-t^{2} B^{\prime} .
$$

Setting $t^{2}=0$ gives the matrix equation

$$
L_{B} E_{L}(\gamma) L_{A}^{-1} R_{A}^{-1} E_{R}(\gamma) R_{B}=I
$$

over the dual numbers $\Lambda[t] /\left(t^{2}\right)$, and this defines $\Phi_{2}(\gamma)$ in $K_{2}\left(\Lambda[t] /\left(t^{2}\right)\right)$.

Then one proves additivity, homotopy invariance with end points fixed, and vanishing whenever $\gamma$ lies in $S S E_{2}\left(\Lambda^{+}\right)$. Homotopy invariance under deformation across a triangle in $S S E(\Lambda)$ uses the purely algebraic Exchange Lemma from [HW], [W7], which is a consequence of the Steinberg relations and which comes from the geometry of two-parameter families of Morse functions. Vanishing under positivity is proved by starting with the expression

$$
\Phi_{2}(\gamma)=\prod x_{i j}(a)
$$

in the Steinberg group $S t\left(\Lambda[t] /\left(t^{2}\right)\right)$ coming from (7.7) and then using the Steinberg relations to reduce it to an expression of the form

$$
\prod_{i>j} x_{i j}(b) \prod_{i<j} x_{i j}(c) .
$$

The reason this can be accomplished is that nonnegativity together with the trace condition $\operatorname{Tr}(A)=\operatorname{Tr}\left(A^{2}\right)=\operatorname{Tr}(B)=\operatorname{Tr}\left(B^{2}\right)=0$ insures expressions of the form $x_{i j}(\alpha) x_{j i}(\beta)$ never obstruct the rearrangement process.

\section{TQFT AND SSE}

P. Gilmer has recently shown in [G2] how the idea of strong shift equivalence over $Z^{+}$and $Q^{+}$arises in topological quantum field theory invariants (e.g., for knots). Related work has been done by D. Silver and S. Williams [SW1], [SW2] more in the context of combinatorial group theory. The setting in [G2] is to start with a pair $(M, \chi)$ where $M$ is a connected manifold of dimension $d+1$ and $\chi$ is a primitive cohomology class in $H^{1}(M)$; i.e., the homomorphism $\chi: H_{1}(M) \rightarrow Z$ is onto. If $M$ has a nonempty boundary (e.g., $M$ is the complement of an open tubular neighborhood of a knot $K^{d-1}$ in $S^{d+1}$ ), assume that $\partial M=K \times S^{1}$ for some (d-1)-manifold $K$ and that $\chi$ restricted to $H_{1}(\partial M)=H_{1}(K) \oplus Z$ is just projection onto the second factor. Select a Seifert surface $\Sigma$ for $(M, \chi)$; namely, $\Sigma=\theta^{-1}(p)$ where $\theta: M \rightarrow S^{1}$ is a smooth function representing $\chi$ and $p$ is not a critical value of $\theta$. Let $M_{\infty}$ denote the infinite cyclic cover of $M$ with respect to the primitive class $\chi$, and let $T$ be the generator of the infinite cyclic group of covering transformations acting on $M_{\infty}$. Lift the Seifert surface $\Sigma$ up to $M_{\infty}$, and consider the cobordism $E$ in $M_{\infty}$ from $\Sigma$ to $T(\Sigma)$ as in Figure 3. 


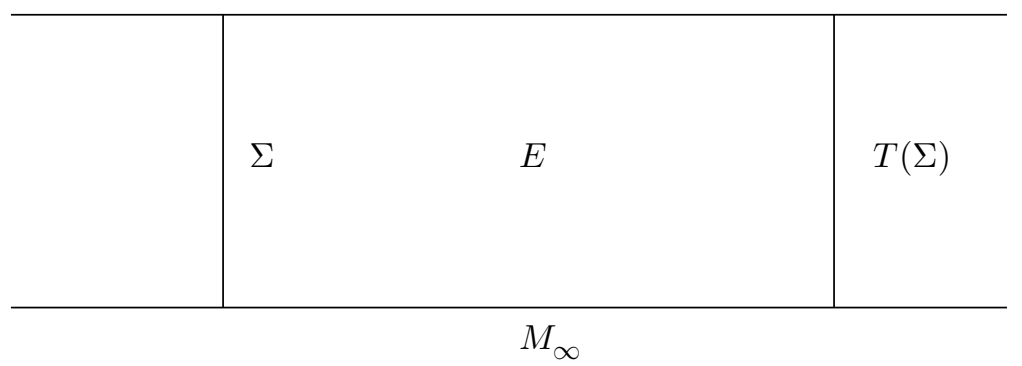

FiguRE 3

For simplicity assume $\partial M$ is empty. Consider the TQFT discussed in $[\mathrm{Q}]$ which takes

$$
V(\Sigma)=Q[\Sigma, B G]
$$

to be vector space over $Q$ with (finite) basis consisting of the set $[\Sigma, B G]$ of homotopy classes of continuous maps from $\Sigma$ to the classifying space $B G$ of the finite group $G$. Select an ordering of the finite set $[\Sigma, B G]$ which has, say, cardinality $n$. The cobordism $E$ from $\Sigma$ to $T(\Sigma)$ produces a homomorphism

$$
Z_{E}: V(\Sigma) \rightarrow V(T(\Sigma))
$$

as in $[Q]$ defined by

$$
Z_{E}([f])=\sum_{[F] \in[E, f ; B G]} \#^{\pi}\left(\operatorname{Map}_{f}(E, B G), F\right)[F \mid T(\Sigma)]
$$

where a representative $f: \Sigma \rightarrow B G$ of each homotopy class $[f]$ of mappings from $\Sigma$ to $B G$ is chosen, where the sum is over the finite set $[E, f ; B G]$ of homotopy classes of maps $F: E \rightarrow B G$ with $F \mid \Sigma=f$, and where the homotopy order is

$$
\#^{\pi}\left(\operatorname{Map}_{f}(E, B G), F\right)=\left(\# \pi_{1}\right)^{-1}\left(\# \pi_{2}\right)\left(\# \pi_{3}\right)^{-1} \cdots .
$$

$\operatorname{Map}_{f}(E, B G)$ is the space of continuous mappings $F: E \rightarrow B G$ with $F \mid \Sigma=f$. The homotopy groups are those of the component of $\operatorname{Map}_{f}(E, B G)$ containing $F$. Each $\pi_{i}$ is a finite set, and $\# \pi_{i}$ denotes the number of elements in $\pi_{i}$. The product is finite because $\operatorname{Map}_{f}(E, B G)$ has only finitely many nontrivial homotopy groups. The homeomorphism $T$ gives an isomorphism from $V(T(\Sigma))$ back to $V(\Sigma)$ by means of which $Z_{E}$ becomes an endomorphism $A=A(\Sigma)$ of $V(\Sigma)$. With respect to the basis for $V(\Sigma)$ chosen above, we see that

$$
A=A(\Sigma) \text { is an } n \times n \text { matrix with coefficients in } Q^{+} .
$$

The rational Turaev-Viro module [G1] is nothing other than the rational dimension group $\left(G_{A} \otimes Q, s_{A}\right)$.

Since $M$ is connected, it is possible to choose a connected Seifert surface $\Sigma$. In this case it follows from (1) of Exercise 4.13 in $[Q]$ that

$$
A(\Sigma) \text { is a matrix with entries in } Z^{+} \text {. }
$$

If $d=0$, then $M=S^{1}, \Sigma$ is a point, and $A=\{1\}$. In [G2] Gilmer proves

Theorem 8.4. If $d \geq 2$, then the strong shift equivalence class of $A(\Sigma)$ over $Z^{+}$is independent of the choice of the connected Seifert surface $\Sigma$ and the choices made in constructing $A(\Sigma)$. 


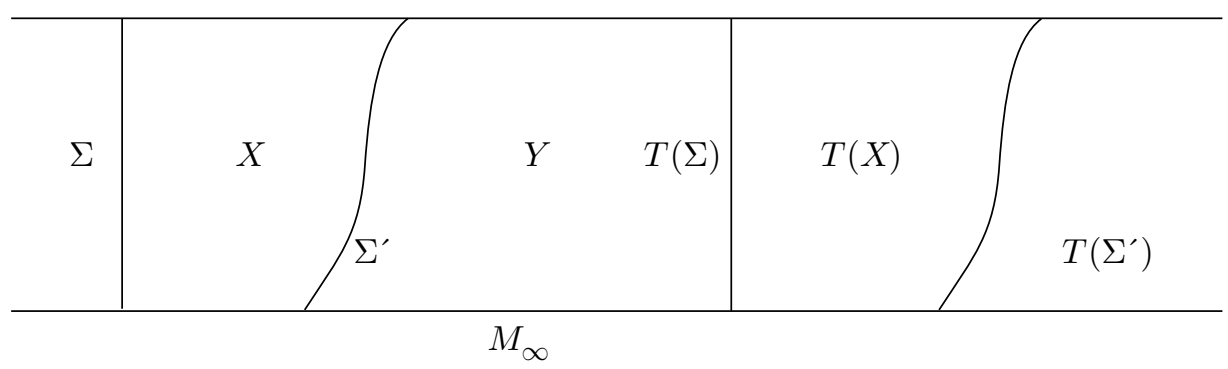

FiguRE 4

The theorem is also true for $d=1$, although Gilmer's proof in this case is different and relies on the elementary topological classification of Riemann surfaces.

Here is Gilmer's idea for showing the strong shift equivalence class of $A(\Sigma)$ is well defined over $Z^{+}$when $d \geq 2$. Let $\Sigma$ and $\Sigma^{\prime}$ be disjoint Seifert surfaces arranged inside $M_{\infty}$ as in Figure 4. The matrix $A=A(\Sigma)$ comes from the cobordism from $\Sigma$ to $T(\Sigma)$. The matrix $A^{\prime}=A\left(\Sigma^{\prime}\right)$ comes from the cobordism from $\Sigma^{\prime}$ to $T\left(\Sigma^{\prime}\right)$. Let $R$ be the matrix obtained by using the formula $(8.1)$ with $T(\Sigma)$ replaced by $\Sigma^{\prime}$ and $E$ replaced by the cobordism $X$ from $\Sigma$ to $\Sigma^{\prime}$. Let $S$ be the matrix obtained by using the formula (8.1) with $\Sigma$ replaced by $\Sigma^{\prime}$ and $E$ replaced by the cobordism $Y$ from $\Sigma^{\prime}$ to $T(\Sigma)$. It follows from the TQFT composition property applied to the unions $X \cup Y$ and $Y \cup T(X)$ that

$$
A=R S \quad \text { and } \quad S R=A^{\prime} .
$$

If $d \geq 1$, Gilmer observes that any two Seifert surfaces for $\chi$ are related by isotopies combined with moves between pairs of disjoint Seifert surfaces as in the diagram above. So the strong shift equivalence class of $A$ over $Q^{+}$is well defined. If $d \geq$ 2 , this can be done in such a way that all the intermediate Seifert surfaces and cobordisms are connected. The argument is like the proof of stable equivalence of Seifert surfaces well known to knot theorists. See [Li, p. 79]. Hence, it follows from (1) of Exercise 4.13 in [Q] that all the strong shift equivalences in (8.5) are over $Z^{+}$ when $d \geq 2$.

A guiding philosophy which led to the development of the $S S E$ spaces is that if objects in a given situation are related to $K_{1}$, then automorphisms should be related to $K_{2}$. In the present context this says the next step beyond [G2] and [SW1], [SW2] is to study the connection between diffeomophisms and symmetries of shifts or between group automorphisms and symmetries of shifts. For example, let $\operatorname{Diff}(M, \chi)$ denote the group of diffeomorphisms of $M$ which preserve $\chi$. Then one expects there to be a homomorphism

$$
\pi_{0} \operatorname{Diff}(M, \chi) \rightarrow \pi_{1}\left(S S E\left(\Lambda^{+}\right), A\right) /\left\{\sigma_{A}^{n}\right\}
$$

or something like it where $\Lambda$ is $Z$ or $Q$. There should be other variations depending on the choice of TQFT. Preliminary work indicates that one way to accomplish this program is by using the $S S E$ spaces together with one and two parameter families of functions from $M$ to $S^{1}$ in a similar fashion to the methods of pseudo-isotopy theory $[\mathrm{C}],[\mathrm{HW}]$. Another approach involves the space of Seifert surfaces, which are analogous to cross-sections of flows and Markov partitions. 


\section{Future PROBLEMS AND PROJECTS}

Number 1 Let $A$ be primitive. Are there only finitely many $S S E$ classes over $Z^{+}$ within the $S S E$ class of $A$ over $Z$ ? This question was asked by Dennis Sullivan in the 1970's and again recently by Doug Lind. If this is not true in general, finding an example where there are infinitely many $S S E$ classes over $Z^{+}$requires new invariants and new methods for producing candidates $A$ and $B$ of matrices which are not SSE over $Z^{+}$.

Number 2 Are the matrices $A_{n}$ and $B_{n}$ of (2.16) strong shift equivalent over $Z^{+}$ for $n \geq 4$ ?

Number 3 Study the algebraic shift equivalence problem. Does $S E$ imply $S S E$ over a commutative regular ring $\Lambda$ ?

Number 4 Study $S S E$ over other rings. Are the $7 \mathrm{x} 7$ counterexamples in Section 5 and [KR5], [KR6] strong shift equivalent over $Q^{+}$or $R^{+}$?

Number 5 For a primitive matrix $A$ does $\operatorname{Image}\left(\delta_{A}\right)$ always have finite index in $\operatorname{Aut}\left(s_{A}\right)$ ? This is similar to Number 1. For simplicity, assume $\operatorname{Aut}\left(s_{A}\right)$ is a finitely generated abelian group, which is the case when $\operatorname{det}(t I-A)$ is irreducible as explained in [BLR].

Number 6 Compare $s g c_{k}$ and $\Phi_{2 m}$. Geller-Roberts show in [GR] that

$$
K_{2}\left(Z[t] /\left(t^{m+1}\right),(t)\right)=\bigoplus_{k=2}^{m+1} Z / k Z .
$$

Kim-Roush show in the Appendix to [W7] that $s g c_{2}=\Phi_{2}$. What is the relationship between $\Phi_{2 m}$ and $s g c_{k}$ for $k=2,3, \ldots, m+1$ ?

Number 7 Looking beyond the classification of subshifts of finite type, there is the general problem of studying the relation between the spaces $S S E\left(\Lambda^{+}\right)$and $\operatorname{SSE}(\Lambda)$.

Here is an example. Let $\Lambda=Z$. Recall that the group $\operatorname{Inert}\left(\sigma_{A}\right)$ of inert automorphisms of $\operatorname{Aut}\left(\sigma_{A}\right)$ is the kernel of the dimension group representation (3.8). In view of (3.5), Aut $\left(s_{A}\right)$ is often a finitely generated abelian group [BLR], and consequently much of the complexity of Aut $\left(\sigma_{A}\right)$ typically lies in $\operatorname{Inert}\left(\sigma_{A}\right)$. For example, if $p$ is prime, then $\operatorname{Aut}\left(s_{p}\right)=Z \oplus\{ \pm 1\}$. Moreover, $s_{p}=s\left(\sigma_{p}\right)$ generates the infinite cyclic summand, and

$$
\operatorname{Aut}\left(\sigma_{p}\right)=Z \oplus \operatorname{Inert}\left(\sigma_{p}\right) .
$$

From the early work of Hedlund and co-workers [H], the (still open) question

$$
\text { Is Inert }\left(\sigma_{p}\right) \text { generated by elements of finite order? by involutions? }
$$

was raised by Frank Rhodes for $p=2$. During the 1980's this was generalized to the Finite Order Generation Conjecture (FOG):

$$
\operatorname{Inert}\left(\sigma_{A}\right) \text { is generated by elements of finite order when } A \text { is primitive. }
$$

The main reason for this conjecture was that historically examples of elements (of finite and infinite order) in $\operatorname{Inert}\left(\sigma_{A}\right)$ had been constructed by the classical "marker method" or ingenious extensions thereof, and therefore these elements were always products of elements of finite order. See [BLR]. The first counterexample to FOG was given in [KRW2], [KRW3] using the method of nonnegative row and column operations on matrices over $Z^{+}[t]$ together with the characterization in $[\mathrm{BF}]$ of finite order inert actions on periodic points. These methods also give counterexamples to FOG for matrices $A$ where $\operatorname{Trace}(A)=\operatorname{Trace}\left(A^{2}\right)=0$ and for higher vanishing 
trace conditions as well. The question is whether there are "natural" homomorphisms of $\operatorname{Inert}\left(\sigma_{A}\right)$ into nontorsion abelian groups which detect these new infinite order elements in Inert $\left(\sigma_{A}\right)$. Here is a proposal. Using (3.6) together with the exact homotopy sequence of the pair $\left(S S E(Z), S S E\left(Z^{+}\right)\right)$yields the isomorphism

$$
\begin{aligned}
\operatorname{Inert}\left(\sigma_{A}\right) / \operatorname{Simp}\left(\sigma_{A}\right) & =\pi_{2}\left(\operatorname{SSE}(Z), \operatorname{SSE}\left(Z^{+}\right) ; A\right) \\
& =\pi_{2}\left(\operatorname{SSE}(Z), S S E_{2}\left(Z^{+}\right) ; A\right)
\end{aligned}
$$

under the condition that $\operatorname{Trace}(A)=\operatorname{Trace}\left(A^{2}\right)=0$. As a result, the discussion in [W7, Section 7 and Section 8] basically goes halfway towards producing a homomorphism

$$
\Theta: \operatorname{Inert}\left(\sigma_{A}\right) \rightarrow K_{3}\left(Z[t] /\left(t^{2}\right),(t)\right) .
$$

The other half would be to show the construction is well defined on homotopy classes. Maybe higher vanishing trace conditions on $A$ are needed for this. An old result of Soulé says that

$$
K_{3}\left(Z[t] /\left(t^{2}\right),(t)\right) \otimes Q=Q
$$

A proof using cyclic homology theory can be found in [Lo, 5.4.17 and Chapter 11]. Does the conjectured $\Theta$ composed with the cyclic homology Chern character [Lo, Chapter 11] detect infinite order elements in $\operatorname{Inert}\left(\sigma_{A}\right)$, at least for certain $A$ ?

Number 8 There is another connection between strong shift equivalence theory and cyclic homology theory. Namely, the SSE spaces are cyclic spaces [Lo, Chapter 7]. An n-cell of $S S E$ is given by an n-tuple $\left(A_{0}, \ldots, A_{n}\right)$ of morphisms (e.g., matrices in the case of $S S E\left(\Lambda^{+}\right)$and $\left.S S E(\Lambda)\right)$ together with elementary strong shift equivalences $\left(R_{i j}, S_{j i}\right): A_{i} \rightarrow A_{j}$ whenever $i<j$ which satisfy the Triangle Identities

$$
\begin{aligned}
& R_{i k}=R_{i j} R_{j k} \\
& S_{j i}=R_{j k} S_{k i} \\
& S_{k j}=S_{k i} R_{i j}
\end{aligned}
$$

for $i<j<k$. Let $R_{0}=S_{n, 0}$ and $R_{i}=R_{i-1, i}$ for $i=1, \ldots, n$. The Triangle Identities show that the simplex is completely determined by the $(\mathrm{n}+1)$-tuple $\left(R_{0}, \ldots, R_{n}\right)$. The cyclic operator $t_{n}$ on $\mathrm{n}$-simplices is given by

$$
t_{n}\left(R_{0}, \ldots, R_{n}\right)=\left(R_{n}, R_{0}, \ldots, R_{n-1}\right) .
$$

It is certainly premature to comment on the significance or ramifications of this observation. But it is interesting that the SSE spaces, which appeared in a totally different context, do turn out to have cyclic structures.

Number 9 Explore the connection between TQFT and SSE. In particular, study the representations (8.6) of $\pi_{0} \operatorname{Diff}(M, \chi)$.

\section{REFERENCES}

[A] R.L. Adler, The torus and the disk, IBM Journal of Research and Development, Vol. 31, No.2, March 1987, 224-234 CMP 19:14

[AKM] R.L. Adler, A. Konheim, and M. McAndrew, Topological entropy, TAMS 114, 1965, 309-319 MR 30:5291

[B] K. Baker, Strong shift equivalence of $2 \times 2$ matrices of non-negative integers, ETDS, 1983, 3, 501-508 MR 86g:28021

[Bl] S. Bloch, Algebraic K-theory and crystalline cohomology, Pub. Math. IHES 47, 1977, 197-238 MR 81j:14011

[Bo] M. Boyle, The stochastic shift equivalence conjecture is false, Contemporary Mathematics, Vol. 135, 1992, 107-110 MR 93i:28013 
[Bow] R. Bowen, Markov partitions for Axiom A diffeomorphisms, Amer. Jour. Math. 92, 1970, 725-747 MR 43:2740

[BF] M. Boyle and U.Fiebig, The action of inert finite order automorphisms on finite subsystems, ETDS 11, 1991, 413-425 MR 92g:58029

[BH1] M. Boyle and D. Handelman, Algebraic shift equivalence and primitive matrices, Trans. AMS 336, No. 1, 1993, 121-149. MR 93e:58050

[BH2] The spectra of nonnegative matrices via symbolic dynamics, Annals of Mathematics, 133, 1991, 249-316 MR 92d:58057

[BK1] M. Boyle and W. Krieger, Periodic points and automorphisms of the shift, Trans. AMS 302, 1987, 125-149 MR 88g:54065

[BK2] , Automorphisms and subsystems of the shift, Jour. Reine Angew. Math. 437, 1993, 13-28 MR 95b:54051

[BLR] M. Boyle, D. Lind, and D. Rudolph, The automorphism group of a shift of finite type, Trans. AMS 306, 1988, 71-114 MR 89m:54051

[BMT] M. Boyle, B. Marcus, and P. Trow, Resolving maps and the dimension group for shifts of finite type, Mem. Amer. Math. Soc. vol. 70 (1987), Number 377, Providence, R.I. MR 89c:28019

[BW] M. Boyle and J.B. Wagoner, Nonnegative algebraic K-theory and subshifts of finite type, in preparation

$[\mathrm{BaW}]$ L. Badoian and J.B. Wagoner, Simple connectivity of the Markov partition space, preprint, UC Berkeley, 1998, to appear in Pac. Jour. Math.

[C] J. Cerf, La stratification naturelle des espaces de fonctions différentiables réelles et le theoreme de la pseudo-isotopie, Publ. Math. IHES, No. 39, 1970, 5-173 MR 45:1176

[CK1] J. Cuntz and W. Krieger, Topological Markov chains and dicyclic dimension groups, J. Reine Angew. Math. 320, 1980, 44-51 MR 81m:54074

[CK2] A class of $C^{*}$-Algebras and topological Markov chains, Inventiones Math. 56, 1980, 251-268 MR 82f:46073a

[E] E.G. Effros, Dimensions and $C^{*}$ Algebras, CBMS No. 46, AMS, 1981 MR 84k:46042

[F] U. Fiebig, Gyration numbers for involutions of subshifts of finite type I, Forum Math. 4, 1992, 77-108; and II, Forum Math.4, 1992, 183-211 MR 93g:58041; MR 93g:58042

[Fr] J. Franks, Homology and dynamical systems, CBMS No. 49, AMS, 1982 MR 84f:58067

[G1] P.M. Gilmer, Invariants for one-dimensional cohomology classes arising from TQFT, Topology and its Applications 75, 1997, 217-259 MR 97k:57018

[G2] Topological quantum field theory and strong shift equivalence, preprint from Louisiana State University, 1997, to appear in Bulletin of the Canadian Mathematical Society

[GR] S. Geller and L. Roberts, $K_{2}$ of some truncated polynomial rings, Ring Theory Waterloo, Springer-Verlag LNM 734, 1979, 249-278 MR 80k:13005

[H] G. Hedlund, Endomorphisms and automorphisms of shift dynamical systems, Math. Systems Theory 3, 1969, 320-375 MR 41:4510

[HW] A. Hatcher and J.B. Wagoner, Pseudo Isotopies of Compact Manifolds, Astérisque No. 6, Société Mathématique de France, 1973 MR 50:5821

[K] B. Kitchens, Symbolic dynamics, one-sided, two-sided and countable state Markov shifts, Springer-Verlag, 1998 MR 98k:58079

[Ke] F. Keune, The relativization of $K_{2}$, Journal of Algebra, 1978, 159-177 MR 80a:18013

[Kol] A.N. Kolomogorov, New metric invariants of transitive dynamical systems and automorphisms of Lebesgue spaces, Dokl. Akad. Nauk. SSSR, 119, 1958, 861-864

[Kr] W. Krieger, On dimension functions and topological Markov chains, Invent. Math. 56, 1980, 239-250 MR 81m:28018

[KOR] K.H. Kim, N. Ormes, and F.W. Roush, The spectra of nonnegative integer matrices via formal power series, preprint, University of Texas at Austin, 1998

[KR1] K.H. Kim and F.W. Roush, Some results on decidability of shift equivalence, J. Comb. Inform. System Sci. 4, 1979, 123-146. MR 81m:58064

[KR2] _ Decidability of shift equivalence, in J. W. Alexander, ed., Dynamical Systems, Lecture Notes in Mathematics, vol. 1342, Springer-Verlag, Heidelberg, 1988 MR 90g:54033

[KR3] On the structure of inert automorphisms of subshifts, Pure Mathematics and Applications 2, 1991, 3-22 MR 94c:58056 
,Williams's conjecture is false for reducible subshifts, Jour. AMS 5, 1992, 213-215 MR 92j:54055

[KR5] Williams's conjecture is false for irreducible subshifts, ERA AMS 3, 1997, 105-109 MR 98i:58081

[KR6] Williams's conjecture is false for irreducible subshifts, preprint from Alabama State University, 1997, to appear in Annals of Mathematics

[KR7] Path components of matrices and strong shift equivalence over $Q^{+}$, Linear Algebra and Applications 145, 1991, 177-186 MR 92c:58027

[KR8] _ Strong shift equivalence of Boolean and positive rational matrices, Linear Algebra and Applications 161, 1992, 153-164 MR 93h:15012

[KRW1] K.H. Kim, F.W. Roush, and J. Wagoner, Automorphisms of the dimension group and gyration numbers, Jour. AMS 5, 1992, 191-212. MR 93h:54026

, Characterization of inert actions on periodic points, Part I and Part II, preprint, UC Berkeley, 1997, to appear in Forum Mathematicum

[KRW3] _ Inert Actions on Periodic Points, ERA AMS 3, 1997, 5562 MR 98i:54021

[Li] W.B.R. Lickorish An Introduction to Knot Theory, GTM 175, Springer-Verlag, 1997 MR 98f:57015

[Lo] J.- L. Loday, Cyclic Homology, Springer-Verlag, Second Edition, 1998 MR 98h:16014

[LM] D. Lind and B. Marcus, An introduction to symbolic dynamics and coding, Cambridge University Press, 1995 MR 97a:58050

[M] B. Marcus, Symbolic dynamics and connections to coding theory, automata theory and system theory, AMS Proc. Symp. Applied Math. 50, 1995, 95-108. MR 97a:58052

[Mi] J. Milnor, Introduction to Algebraic K-Theory, Annals of Mathematics Studies Number 72, Princeton University Press, 1971 MR 50:2304

[MB] S. MacLane and G. Birkhoff, Algebra, Macmillan Company, Second Edition, 1979 MR 80d:00002

[MH] M. Morse and G.A. Hedlund, Symbolic Dynamics, Amer. Jour. Math. 60 (1938), 815-866.

[MS] H. Maazen and J. Stienstra, A presentation for $K_{2}$ of split radical pairs, Journal of Pure and Applied Algebra 10, 1977, 271-294 MR 57:12485

[MT1] B. Marcus and S. Tuncel, Entropy at a weight-per-symbol and an imbedding theorem for Markov chains, Inventiones Mathematicae 102, 1990, 235-266. MR 91k:28023

[MT2] , The weight-per-symbol polytope and scaffolds of invariants associated with Markov chains, Ergodic Theory and Dynamical Systems 11, 1991, 129-180. MR 92g:28038

[MT3] Matrices of polynomials, positivity, and finite equivalence of Markov chains, AMS Journal 6, 1993, 131-147 MR 93e:28022

[N] M. Nasu, Topological conjugacy for sofic systems and extensions of automorphisms of finite subsystems of topological Markov shifts, Springer Lecture Notes in Math 1342, 1988 MR 89j:54045

[PT] W. Parry and S. Tuncel, Classification problems in ergodic theory, LMS Lecture Notes 67, Cambridge University Press, 1982 MR 84g:28024

[PW] W. Parry and R.F. Williams, Block coding and a zeta function for Markov chains, Proc. LMS 35, 1977, 483-495 MR 57:6368

[Q] F. Quinn, Lectures on axiomatic topological quantum field theory, Geometry and Quantum Field Theory (edited by D. Freed and K. Uhlenbeck), IAS/Park City Mathematics Series, Vol. 1, AMS, 1995, 325-453 MR 96e:57021

[R] J. Rosenberg, Algebraic K-Theory and Its Applications, GTM 147, Springer-Verlag, 1994 MR 95e:19001

[S] S. Smale, Differential Dynamical Systems, BAMS, Vol. 73, No. 6, 1967, 747-847 MR 37:3598

[Sh] C. Shannon, A mathematical theory of communication, Bell Sys. Tech Jour. 27, 1948, 379-423, 623-656 MR 10:133e

[St] J. Stienstra, On $K_{2}$ and $K_{3}$ of truncated polynomial rings, LNM 854, Springer, 1981 MR 82k: 13016

[SW1] D. Silver and S. Williams, Augmented group systems and shifts of finite type, Israel Journal of Mathematics 95, 1996, 231-251 MR 98b:20045 
[SW2] Knot invariants from symbolic dynamical systems, to appear in Trans. AMS 351, No. 8, 1999, 3243-3265 CMP 97:17

[T] S. Tuncel, Faces of Markov chains and matrices of polynomials, Contemporary Mathematics, Vol. 135, AMS, 1992, 391-422 MR 94m:28034

[vdK] W. van der Kallen, Le $K_{2}$ des nombres duaux, C.R. Acad. Sc. Paris, Série A, t. 273 , 20 décembre 1971, 1204-1207 MR 45:252

[W1] J.B. Wagoner, Markov partitions and $K_{2}$, Publ. Math. IHES, No. 65, 1987, 91-129 MR 90d:28022

[W2] Triangle identities and symmetries of a subshift of finite type, Pacific Jour. Math. 144, 1990, 181-205 MR 91h:28017

[W3] Higher dimensional shift equivalence is the same as strong shift equivalence over the integers, Proc. AMS 109, 1990, 527-536 MR 90i:54088

[W4] Eventual finite order generation for the kernel of the dimension group representation, Trans. AMS 317, 1990, 331-350 MR 91a:54055

[W5] Classification of subshifts of finite type revisited, Contemporary Mathematics, Vol. 135, AMS, 1992, 423-436 MR 93m:20004

[W6] Markov chains, $C^{*}$-algebras, and $K_{2}$, Advances of Mathematics, Vol. 71, No. 2, 1988, 133-185 MR 90j:46059

[W7] Strong shift equivalence and $K_{2}$ of the dual numbers, preprint from UC Berkeley, 1998, to appear in Jour. Reine Angew. Math.

[Wi1] R.F. Williams, Classification of subshifts of finite type, Annals of Math.(2) 98, 1973, 120-153; Errata ibid. 99, 1974, 380-381. MR 48:9769

[Wi2] Shift equivalence of matrices in GL(2,Z), Cont. Math., Vol. 135, AMS, 1992, 445-451 MR 93k:58183

[Wi3] Classification of 1-dimensional attractors, Global Analysis, Proceedings of the Symposium on Pure Mathematics 14, AMS, 1970, 341-361 MR 42:1134

[Wi4] Addendum to "Classification of 1-dimensional attractors", preprint from University of Texas, Austin, 1998

Mathematics, University of California, Berkeley, CA 94720

E-mail address: wagoner@math.berkeley.edu 